\title{
Accelerated Proximal Stochastic Dual Coordinate Ascent for Regularized Loss Minimization
}

\author{
Shai Shalev-Shwartz* Tong Zhang ${ }^{\dagger \ddagger}$
}

\begin{abstract}
We introduce a proximal version of the stochastic dual coordinate ascent method and show how to accelerate the method using an inner-outer iteration procedure. We analyze the runtime of the framework and obtain rates that improve state-of-the-art results for various key machine learning optimization problems including SVM, logistic regression, ridge regression, Lasso, and multiclass SVM. Experiments validate our theoretical findings.
\end{abstract}

\section{Introduction}

We consider the following generic optimization problem associated with regularized loss minimization of linear predictors: Let $X_{1}, \ldots, X_{n}$ be matrices in $\mathbb{R}^{d \times k}$ (referred to as instances), let $\phi_{1}, \ldots, \phi_{n}$ be a sequence of vector convex functions defined on $\mathbb{R}^{k}$ (referred to as loss functions), let $g(\cdot)$ be a convex function defined on $\mathbb{R}^{d}$ (referred to as a regularizer), and let $\lambda \geq 0$ (referred to as a regularization parameter). Our goal is to solve:

$$
\min _{w \in \mathbb{R}^{d}} P(w) \quad \text { where } P(w)=\left[\frac{1}{n} \sum_{i=1}^{n} \phi_{i}\left(X_{i}^{\top} w\right)+\lambda g(w)\right] .
$$

For example, in ridge regression the regularizer is $g(w)=\frac{1}{2}\|w\|_{2}^{2}$, the instances are column vectors, and for every $i$ the $i$ 'th loss function is $\phi_{i}(a)=\frac{1}{2}\left(a-y_{i}\right)^{2}$, for some scalar $y_{i}$.

Let $w^{*}=\operatorname{argmin}_{w} P(w)$ (we will later make assumptions that imply that $w^{*}$ is unique). We say that $w$ is $\epsilon$-accurate if $P(w)-P\left(w^{*}\right) \leq \epsilon$. Our main result is a new algorithm for solving (1). If $g$ is 1-strongly convex and each $\phi_{i}$ is $(1 / \gamma)$-smooth (meaning that its gradient is $(1 / \gamma)$-Lipschitz), then our algorithm finds, with probability of at least $1-\delta$, an $\epsilon$-accurate solution to (1) in time

$$
\begin{aligned}
& O\left(d\left(n+\min \left\{\frac{1}{\lambda \gamma}, \sqrt{\frac{n}{\lambda \gamma}}\right\}\right) \log (1 / \epsilon) \log (1 / \delta) \max \left\{1, \log ^{2}(1 /(\lambda \gamma n))\right\}\right) \\
& =\tilde{O}\left(d\left(n+\min \left\{\frac{1}{\lambda \gamma}, \sqrt{\frac{n}{\lambda \gamma}}\right\}\right)\right) .
\end{aligned}
$$

This applies, for example, to ridge regression and to logistic regression with $L_{2}$ regularization. The $O$ notation hides constants terms and the $\tilde{O}$ notation hides constants and logarithmic terms. We make these explicit in the formal statement of our theorems.

${ }^{*}$ School of Computer Science and Engineering, The Hebrew University, Jerusalem, Israel

${ }^{\dagger}$ Department of Statistics, Rutgers University, NJ, USA

${ }^{\ddagger}$ Baidu Inc., Beijing, China 
Intuitively, we can think of $\frac{1}{\lambda \gamma}$ as the condition number of the problem. If the condition number is $O(n)$ then our runtime becomes $\tilde{O}(d n)$. This means that the runtime is nearly linear in the data size. This matches the recent result of Shalev-Shwartz and Zhang [25], Le Roux et al. [15], but our setting is significantly more general. When the condition number is much larger than $n$, our runtime becomes $\tilde{O}\left(d \sqrt{\frac{n}{\lambda \gamma}}\right)$. This significantly improves over the result of [25, 15]. It also significantly improves over the runtime of accelerated gradient descent due to Nesterov [18], which is $\tilde{O}\left(d n \sqrt{\frac{1}{\lambda \gamma}}\right)$.

By applying a smoothing technique to $\phi_{i}$, we also derive a method that finds an $\epsilon$-accurate solution to (1) assuming that each $\phi_{i}$ is $O(1)$-Lipschitz, and obtain the runtime

$$
\tilde{O}\left(d\left(n+\min \left\{\frac{1}{\lambda \epsilon}, \sqrt{\frac{n}{\lambda \epsilon}}\right\}\right)\right) .
$$

This applies, for example, to SVM with the hinge-loss. It significantly improves over the rate $\frac{d}{\lambda \epsilon}$ of SGD (e.g. [22]), when $\frac{1}{\lambda \epsilon} \gg n$.

We can also apply our results to non-strongly convex regularizers (such as the $L_{1}$ norm regularizer), or to non-regularized problems, by adding a slight $L_{2}$ regularization. For example, for $L_{1}$ regularized problems, and assuming that each $\phi_{i}$ is $(1 / \gamma)$-smooth, we obtain the runtime of

$$
\tilde{O}\left(d\left(n+\min \left\{\frac{1}{\epsilon \gamma}, \sqrt{\frac{n}{\epsilon \gamma}}\right\}\right)\right) .
$$

This applies, for example, to the Lasso problem, in which the goal is to minimize the squared loss plus an $L_{1}$ regularization term.

To put our results in context, in the table below we specify the runtime of various algorithms (while ignoring constants and logarithmic terms) for three key machine learning applications; SVM in which $\phi_{i}(a)=\max \{0,1-a\}$ and $g(w)=\frac{1}{2}\|w\|_{2}^{2}$, Lasso in which $\phi_{i}(a)=\frac{1}{2}\left(a-y_{i}\right)^{2}$ and $g(w)=\sigma\|w\|_{1}$, and Ridge Regression in which $\phi_{i}(a)=\frac{1}{2}\left(a-y_{i}\right)^{2}$ and $g(w)=\frac{1}{2}\|w\|_{2}^{2}$. Additional applications, and a more detailed runtime comparison to previous work, are given in Section 5. In the table below, SGD stands for Stochastic Gradient Descent, and AGD stands for Accelerated Gradient Descent.

\begin{tabular}{c|c|c}
\hline Problem & Algorithm & Runtime \\
\hline \multirow{2}{*}{ SVM } & SGD [22] & $\frac{d}{\lambda \epsilon}$ \\
& AGD [17] & $d n \sqrt{\frac{1}{\lambda \epsilon}}$ \\
& This paper & $d\left(n+\min \left\{\frac{1}{\lambda \epsilon}, \sqrt{\frac{n}{\lambda \epsilon}}\right\}\right)$ \\
\hline \multirow{2}{*}{ Lasso } & Stochastic Coordinate Descent [23, 16] & $\frac{d}{\epsilon^{2}}$ \\
& FISTA [18, 2] & $\frac{d n}{\epsilon}$ \\
& This paper & $d n \sqrt{\frac{1}{\epsilon}}$ \\
Ridge Regression & Exact & $d\left(n+\min \left\{\frac{1}{\epsilon}, \sqrt{\frac{n}{\epsilon}}\right\}\right)$ \\
\hline & SGD [15], SDCA [25] & $d^{2} n+d^{3}$ \\
& AGD [18] & $d\left(n+\frac{1}{\lambda}\right)$ \\
& This paper & $d n \sqrt{\frac{1}{\lambda}}$ \\
& & $d\left(n+\min \left\{\frac{1}{\lambda}, \sqrt{\frac{n}{\lambda}}\right\}\right)$ \\
\hline
\end{tabular}


Technical contribution: Our algorithm combines two ideas. The first is a proximal version of stochastic dual coordinate ascent (SDCA) 1 In particular, we generalize the recent analysis of [25] in two directions. First, we allow the regularizer, $g$, to be a general strongly convex function (and not necessarily the squared Euclidean norm). This allows us to consider non-smooth regularization function, such as the $L_{1}$ regularization. Second, we allow the loss functions, $\phi_{i}$, to be vector valued functions which are smooth (or Lipschitz) with respect to a general norm. This generalization is useful in multiclass applications. As in [25], the runtime of this procedure is $\tilde{O}\left(d\left(n+\frac{1}{\lambda \gamma}\right)\right)$. This would be a nearly linear time (in the size of the data) if $\frac{1}{\lambda \gamma}=O(n)$. Our second idea deals with the case $\frac{1}{\lambda \gamma} \gg n$ by iteratively approximating the objective function $P$ with objective functions that have a stronger regularization. In particular, each iteration of our acceleration procedure involves approximate minimization of $P(w)+\frac{\kappa}{2}\|w-y\|_{2}^{2}$, with respect to $w$, where $y$ is a vector obtained from previous iterates and $\kappa$ is order of $1 /(\gamma n)$. The idea is that the addition of the relatively strong regularization makes the runtime of our proximal stochastic dual coordinate ascent procedure be $\tilde{O}(d n)$. And, with a proper choice of $y$ at each iteration, we show that the sequence of solutions of the problems with the added regularization converge to the minimum of $P$ after $\sqrt{\frac{1}{\lambda \gamma n}}$ iterations. This yields the overall runtime of $d \sqrt{\frac{n}{\lambda \gamma}}$.

Additional related work: As mentioned before, our first contribution is a proximal version of the stochastic dual coordinate ascent method and extension of the analysis given in Shalev-Shwartz and Zhang [25]. Stochastic dual coordinate ascent has also been studied in Collins et al. [3] but in more restricted settings than the general problem considered in this paper. One can also apply the analysis of stochastic coordinate descent methods given in Richtárik and Takáč [19] on the dual problem. However, here we are interested in understanding the primal sub-optimality, hence an analysis which only applies to the dual problem is not sufficient.

The generality of our approach allows us to apply it for multiclass prediction problems. We discuss this in detail later on in Section 5. Recently, [13] derived a stochastic coordinate ascent for structural SVM based on the Frank-Wolfe algorithm. Although with different motivations, for the special case of multiclass problems with the hinge-loss, their algorithm ends up to be the same as our proximal dual ascent algorithm (with the same rate). Our approach allows to accelerate the method and obtain an even faster rate.

The proof of our acceleration method adapts Nesterov's estimation sequence technique, studied in Devolder et al. [7], Schmidt et al. [20], to allow approximate and stochastic proximal mapping. See also [1, 6]. In particular, it relies on similar ideas as in Proposition 4 of [20]. However, our specific requirement is different, and the proof presented here is different and significantly simpler than that of [20].

There have been several attempts to accelerate stochastic optimization algorithms. See for example [12, 11, 4] and the references therein. However, the runtime of these methods have a polynomial dependence on $1 / \epsilon$ even if $\phi_{i}$ are smooth and $g$ is $\lambda$-strongly convex, as opposed to the logarithmic dependence on $1 / \epsilon$ obtained here. As in [15, 25], we avoid the polynomial dependence on $1 / \epsilon$ by allowing more than a single pass over the data.

\footnotetext{
${ }^{1}$ Technically speaking, it may be more accurate to use the term randomized dual coordinate ascent, instead of stochastic dual coordinate ascent. This is because our algorithm makes more than one pass over the data, and therefore cannot work directly on distributions with infinite support. However, following the convention in the prior machine learning literature, we do not make this distinction.
} 


\section{Preliminaries}

All the functions we consider in this paper are proper convex functions over a Euclidean space. We use $\mathbb{R}$ to denote the set of real numbers and to simplify our notation, when we use $\mathbb{R}$ to denote the range of a function $f$ we in fact allow $f$ to output the value $+\infty$.

Given a function $f: \mathbb{R}^{d} \rightarrow \mathbb{R}$ we denote its conjugate function by

$$
f^{*}(y)=\sup _{x}\left[y^{\top} x-f(x)\right] .
$$

Given a norm $\|\cdot\|_{P}$ we denote the dual norm by $\|\cdot\|_{D}$ where

$$
\|y\|_{D}=\sup _{x:\|x\|_{P}=1} y^{\top} x .
$$

We use $\|\cdot\|$ or $\|\cdot\|_{2}$ to denote the $L_{2}$ norm, $\|x\|=x^{\top} x$. We also use $\|x\|_{1}=\sum_{i}\left|x_{i}\right|$ and $\|x\|_{\infty}=\max _{i}\left|x_{i}\right|$. The operator norm of a matrix $X$ with respect to norms $\|\cdot\|_{P},\|\cdot\|_{P^{\prime}}$ is defined as

$$
\|X\|_{P \rightarrow P^{\prime}}=\sup _{u:\|u\|_{P=1}}\|X u\|_{P^{\prime}} .
$$

A function $f: \mathbb{R}^{k} \rightarrow \mathbb{R}^{d}$ is $L$-Lipschitz with respect to a norm $\|\cdot\|_{P}$, whose dual norm is $\|\cdot\|_{D}$, if for all $a, b \in \mathbb{R}^{d}$, we have

$$
\|f(a)-f(b)\|_{D} \leq L\|a-b\|_{P} .
$$

A function $f: \mathbb{R}^{d} \rightarrow \mathbb{R}$ is $(1 / \gamma)$-smooth with respect to a norm $\|\cdot\|_{P}$ if it is differentiable and its gradient is $(1 / \gamma)$-Lipschitz with respect to $\|\cdot\|_{P}$. An equivalent condition is that for all $a, b \in \mathbb{R}^{d}$, we have

$$
f(a) \leq f(b)+\nabla f(b)^{\top}(a-b)+\frac{1}{2 \gamma}\|a-b\|_{P}^{2} .
$$

A function $f: \mathbb{R}^{d} \rightarrow \mathbb{R}$ is $\gamma$-strongly convex with respect to $\|\cdot\|_{P}$ if

$$
f(w+v) \geq f(w)+\nabla f(w)^{\top} v+\frac{\gamma}{2}\|v\|_{P}^{2} .
$$

It is well known that $f$ is $\gamma$-strongly convex with respect to $\|\cdot\|_{P}$ if and only if $f^{*}$ is $(1 / \gamma)$-smooth with respect to the dual norm, $\|\cdot\|_{D}$.

The dual problem of (1) is

$$
\max _{\alpha \in \mathbb{R}^{k \times n}} D(\alpha) \text { where } D(\alpha)=\left[\frac{1}{n} \sum_{i=1}^{n}-\phi_{i}^{*}\left(-\alpha_{i}\right)-\lambda g^{*}\left(\frac{1}{\lambda n} \sum_{i=1}^{n} X_{i} \alpha_{i}\right)\right],
$$

where $\alpha_{i}$ is the $i$ 'th column of the matrix $\alpha$, which forms a vector in $\mathbb{R}^{k}$.

We will assume that $g$ is strongly convex which implies that $g^{*}(\cdot)$ is continuous differentiable. If we define

$$
v(\alpha)=\frac{1}{\lambda n} \sum_{i=1}^{n} X_{i} \alpha_{i} \quad \text { and } \quad w(\alpha)=\nabla g^{*}(v(\alpha))
$$

then it is known that $w\left(\alpha^{*}\right)=w^{*}$, where $\alpha^{*}$ is an optimal solution of (2). It is also known that $P\left(w^{*}\right)=$ $D\left(\alpha^{*}\right)$ which immediately implies that for all $w$ and $\alpha$, we have $P(w) \geq D(\alpha)$, and hence the duality gap defined as

$$
P(w(\alpha))-D(\alpha)
$$

can be regarded as an upper bound on both the primal sub-optimality, $P(w(\alpha))-P\left(w^{*}\right)$, and on the dual sub-optimality, $D\left(\alpha^{*}\right)-D(\alpha)$. 


\section{Main Results}

In this section we describe our algorithms and their analysis. We start in Section 3.1 with a description of our proximal stochastic dual coordinate ascent procedure (Prox-SDCA). Then, in Section 3.2 we show how to accelerate the method by calling Prox-SDCA on a sequence of problems with a strong regularization. Throughout the first two sections we assume that the loss functions are smooth. Finally, we discuss the case of Lipschitz loss functions in Section 3.3 .

The proofs of the main acceleration theorem (Theorem 3 ) is given in Section 4 . The rest of the proofs are provided in the appendix.

\subsection{Proximal Stochastic Dual Coordinate Ascent}

We now describe our proximal stochastic dual coordinate ascent procedure for solving (1). Our results in this subsection holds for $g$ being a 1-strongly convex function with respect to some norm $\|\cdot\|_{P^{\prime}}$ and every $\phi_{i}$ being a $(1 / \gamma)$-smooth function with respect to some other norm $\|\cdot\|_{P}$. The corresponding dual norms are denoted by $\|\cdot\|_{D^{\prime}}$ and $\|\cdot\|_{D}$ respectively.

The dual objective in (2) has a different dual vector associated with each example in the training set. At each iteration of dual coordinate ascent we only allow to change the $i$ 'th column of $\alpha$, while the rest of the dual vectors are kept intact. We focus on a randomized version of dual coordinate ascent, in which at each round we choose which dual vector to update uniformly at random.

At step $t$, let $v^{(t-1)}=(\lambda n)^{-1} \sum_{i} X_{i} \alpha_{i}^{(t-1)}$ and let $w^{(t-1)}=\nabla g^{*}\left(v^{(t-1)}\right)$. We will update the $i$-th dual variable $\alpha_{i}^{(t)}=\alpha_{i}^{(t-1)}+\Delta \alpha_{i}$, in a way that will lead to a sufficient increase of the dual objective. For the primal problem, this would lead to the update $v^{(t)}=v^{(t-1)}+(\lambda n)^{-1} X_{i} \Delta \alpha_{i}$, and therefore $w^{(t)}=\nabla g^{*}\left(v^{(t)}\right)$ can also be written as

$$
w^{(t)}=\underset{w}{\operatorname{argmax}}\left[w^{\top} v^{(t)}-g(w)\right]=\underset{w}{\operatorname{argmin}}\left[-w^{\top}\left(n^{-1} \sum_{i=1}^{n} X_{i} \alpha_{i}^{(t)}\right)+\lambda g(w)\right] .
$$

Note that this particular update is rather similar to the update step of proximal-gradient dual-averaging method (see for example Xiao [27]). The difference is on how $\alpha^{(t)}$ is updated.

The goal of dual ascent methods is to increase the dual objective as much as possible, and thus the optimal way to choose $\Delta \alpha_{i}$ would be to maximize the dual objective, namely, we shall let

$$
\Delta \alpha_{i} \underset{\Delta \alpha_{i} \in \mathbb{R}^{k}}{\operatorname{argmax}}\left[-\frac{1}{n} \phi_{i}^{*}\left(-\left(\alpha_{i}+\Delta \alpha_{i}\right)\right)-\lambda g^{*}\left(v^{(t-1)}+(\lambda n)^{-1} X_{i} \Delta \alpha_{i}\right)\right] .
$$

However, for a complex $g^{*}(\cdot)$, this optimization problem may not be easy to solve. To simplify the optimization problem we can rely on the smoothness of $g^{*}$ (with respect to a norm $\|\cdot\|_{D^{\prime}}$ ) and instead of directly maximizing the dual objective function, we try to maximize the following proximal objective which is a lower bound of the dual objective:

$$
\begin{aligned}
\underset{\Delta \alpha_{i} \in \mathbb{R}^{k}}{\operatorname{argmax}}\left[-\frac{1}{n} \phi_{i}^{*}\left(-\left(\alpha_{i}+\Delta \alpha_{i}\right)\right)-\lambda\left(\nabla g^{*}\left(v^{(t-1)}\right)^{\top}(\lambda n)^{-1} X_{i} \Delta \alpha_{i}+\frac{1}{2}\left\|(\lambda n)^{-1} X_{i} \Delta \alpha_{i}\right\|_{D^{\prime}}^{2}\right)\right] \\
=\underset{\Delta \alpha_{i} \in \mathbb{R}^{k}}{\operatorname{argmax}}\left[-\phi_{i}^{*}\left(-\left(\alpha_{i}+\Delta \alpha_{i}\right)\right)-w^{(t-1) \top} X_{i} \Delta \alpha_{i}-\frac{1}{2 \lambda n}\left\|X_{i} \Delta \alpha_{i}\right\|_{D^{\prime}}^{2}\right] .
\end{aligned}
$$


In general, this optimization problem is still not necessarily simple to solve because $\phi^{*}$ may also be complex. We will thus also propose alternative update rules for $\Delta \alpha_{i}$ of the form $\Delta \alpha_{i}=s\left(-\nabla \phi_{i}\left(X_{i}^{\top} w^{(t-1)}\right)-\alpha_{i}^{(t-1)}\right)$ for an appropriately chosen step size parameter $s>0$. Our analysis shows that an appropriate choice of $s$ still leads to a sufficient increase in the dual objective.

It should be pointed out that we can always pick $\Delta \alpha_{i}$ so that the dual objective is non-decreasing. In fact, if for a specific choice of $\Delta \alpha_{i}$, the dual objective decreases, we may simply set $\Delta \alpha_{i}=0$. Therefore throughout the proof we will assume that the dual objective is non-decreasing whenever needed.

The theorems below provide upper bounds on the number of iterations required by our prox-SDCA procedure.

Theorem 1. Consider Procedure Prox-SDCA as given in Figure 1 Let $\alpha^{*}$ be an optimal dual solution and let $\epsilon>0$. For every $T$ such that

$$
T \geq\left(n+\frac{R^{2}}{\lambda \gamma}\right) \log \left(\left(n+\frac{R^{2}}{\lambda \gamma}\right) \cdot \frac{D\left(\alpha^{*}\right)-D\left(\alpha^{(0)}\right)}{\epsilon}\right),
$$

we are guaranteed that $\mathbb{E}\left[P\left(w^{(T)}\right)-D\left(\alpha^{(T)}\right)\right] \leq \epsilon$. Moreover, for every $T$ such that

$$
T \geq\left(n+\left\lceil\frac{R^{2}}{\lambda \gamma}\right\rceil\right) \cdot\left(1+\log \left(\frac{D\left(\alpha^{*}\right)-D\left(\alpha^{(0)}\right)}{\epsilon}\right)\right),
$$

let $T_{0}=T-n-\left\lceil\frac{R^{2}}{\lambda \gamma}\right\rceil$, then we are guaranteed that $\mathbb{E}[P(\bar{w})-D(\bar{\alpha})] \leq \epsilon$.

We next give bounds that hold with high probability.

Theorem 2. Consider Procedure Prox-SDCA as given in Figure 1 Let $\alpha^{*}$ be an optimal dual solution, let $\epsilon_{D}, \epsilon_{P}>0$, and let $\delta \in(0,1)$.

1. For every $T$ such that

$$
T \geq\left\lceil\left(n+\frac{R^{2}}{\lambda \gamma}\right) \log \left(\frac{2\left(D\left(\alpha^{*}\right)-D\left(\alpha^{(0)}\right)\right)}{\epsilon_{D}}\right)\right\rceil \cdot\left\lceil\log _{2}\left(\frac{1}{\delta}\right)\right\rceil,
$$

we are guaranteed that with probability of at least $1-\delta$ it holds that $D\left(\alpha^{*}\right)-D\left(\alpha^{(T)}\right) \leq \epsilon_{D}$.

2. For every $T$ such that

$$
T \geq\left\lceil\left(n+\frac{R^{2}}{\lambda \gamma}\right)\left(\log \left(n+\frac{R^{2}}{\lambda \gamma}\right)+\log \left(\frac{2\left(D\left(\alpha^{*}\right)-D\left(\alpha^{(0)}\right)\right)}{\epsilon_{P}}\right)\right)\right\rceil \cdot\left\lceil\log _{2}\left(\frac{1}{\delta}\right)\right\rceil,
$$

we are guaranteed that with probability of at least $1-\delta$ it holds that $P\left(w^{(T)}\right)-D\left(\alpha^{(T)}\right) \leq \epsilon_{P}$.

3. Let $T$ be such that

$$
T \geq\left(n+\left\lceil\frac{R^{2}}{\lambda \gamma}\right\rceil\right) \cdot\left(1+\left\lceil\log \left(\frac{2\left(D\left(\alpha^{*}\right)-D\left(\alpha^{(0)}\right)\right)}{\epsilon_{P}}\right)\right\rceil\right) \cdot\left\lceil\log _{2}\left(\frac{2}{\delta}\right)\right\rceil,
$$

and let $T_{0}=T-n-\left\lceil\frac{R^{2}}{\lambda \gamma}\right\rceil$. Suppose we choose $\left\lceil\log _{2}(2 / \delta)\right\rceil$ values of $t$ uniformly at random from $T_{0}+1, \ldots, T$, and then choose the single value of $t$ from these $\left\lceil\log _{2}(2 / \delta)\right\rceil$ values for which $P\left(w^{(t)}\right)-D\left(\alpha^{(t)}\right)$ is minimal. Then, with probability of at least $1-\delta$ we have that $P\left(w^{(t)}\right)-D\left(\alpha^{(t)}\right) \leq$ $\epsilon_{P}$. 


\section{Procedure Proximal Stochastic Dual Coordinate Ascent: Prox-SDCA $\left(P, \epsilon, \alpha^{(0)}\right)$}

Goal: Minimize $P(w)=\frac{1}{n} \sum_{i=1}^{n} \phi_{i}\left(X_{i}^{\top} w\right)+\lambda g(w)$

Input: Objective $P$, desired accuracy $\epsilon$, initial dual solution $\alpha^{(0)}\left(\right.$ default: $\alpha^{(0)}=0$ ) Assumptions:

$\forall i, \phi_{i}$ is $(1 / \gamma)$-smooth w.r.t. $\|\cdot\|_{P}$ and let $\|\cdot\|_{D}$ be the dual norm of $\|\cdot\|_{P}$ $g$ is 1-strongly convex w.r.t. $\|\cdot\|_{P^{\prime}}$ and let $\|\cdot\|_{D^{\prime}}$ be the dual norm of $\|\cdot\|_{P^{\prime}}$ $\forall i,\left\|X_{i}\right\|_{D \rightarrow D^{\prime}} \leq R$

Initialize $v^{(0)}=\frac{1}{\lambda n} \sum_{i=1}^{n} X_{i} \alpha_{i}^{(0)}, w^{(0)}=\nabla g^{*}(0)$

Iterate: for $t=1,2, \ldots$

Randomly pick $i$

Find $\Delta \alpha_{i}$ using any of the following options

(or any other update that achieves a larger dual objective):

\section{Option I:}

$$
\Delta \alpha_{i}=\underset{\Delta \alpha_{i}}{\operatorname{argmax}}\left[-\phi_{i}^{*}\left(-\left(\alpha_{i}^{(t-1)}+\Delta \alpha_{i}\right)\right)-w^{(t-1)^{\top}} X_{i} \Delta \alpha_{i}-\frac{1}{2 \lambda n}\left\|X_{i} \Delta \alpha_{i}\right\|_{D^{\prime}}^{2}\right]
$$

\section{Option II:}

Let $u=-\nabla \phi_{i}\left(X_{i}^{\top} w^{(t-1)}\right)$ and $q=u-\alpha_{i}^{(t-1)}$

$$
\text { Let } s=\underset{s \in[0,1]}{\operatorname{argmax}}\left[-\phi_{i}^{*}\left(-\left(\alpha_{i}^{(t-1)}+s q\right)\right)-s w^{(t-1)^{\top}} X_{i} q-\frac{s^{2}}{2 \lambda n}\left\|X_{i} q\right\|_{D^{\prime}}^{2}\right]
$$

Set $\Delta \alpha_{i}=s q$

\section{Option III:}

Same as Option II but replace the definition of $s$ as follows:

Let $s=\min \left(1, \frac{\phi_{i}\left(X_{i}^{\top} w^{(t-1)}\right)+\phi_{i}^{*}\left(-\alpha_{i}^{(t-1)}\right)+w^{(t-1)^{\top}} X_{i} \alpha_{i}^{(t-1)}+\frac{\gamma}{2}\|q\|_{D}^{2}}{\|q\|_{D}^{2}\left(\gamma+\frac{1}{\lambda n}\left\|X_{i}\right\|_{D \rightarrow D^{\prime}}^{2}\right)}\right)$

\section{Option IV:}

Same as Option III but replace $\left\|X_{i}\right\|_{D \rightarrow D^{\prime}}^{2}$ in the definition of $s$ with $R^{2}$

\section{Option V:}

Same as Option II but replace the definition of $s$ to be $s=\frac{\lambda n \gamma}{R^{2}+\lambda n \gamma}$

$\alpha_{i}^{(t)} \leftarrow \alpha_{i}^{(t-1)}+\Delta \alpha_{i}$ and for $j \neq i, \alpha_{j}^{(t)} \leftarrow \alpha_{j}^{(t-1)}$

$v^{(t)} \leftarrow v^{(t-1)}+(\lambda n)^{-1} X_{i} \Delta \alpha_{i}$

$w^{(t)} \leftarrow \nabla g^{*}\left(v^{(t)}\right)$

\section{Stopping condition:}

Let $T_{0}<t$ (default: $T_{0}=t-n-\left\lceil\frac{R^{2}}{\lambda \gamma}\right\rceil$ )

Averaging option:

Let $\bar{\alpha}=\frac{1}{t-T_{0}} \sum_{i=T_{0}+1}^{t} \alpha^{(i-1)}$ and $\bar{w}=\frac{1}{t-T_{0}} \sum_{i=T_{0}+1}^{t} w^{(i-1)}$

\section{Random option:}

Let $\bar{\alpha}=\alpha^{(i)}$ and $\bar{w}=w^{(i)}$ for some random $i \in T_{0}+1, \ldots, t$

Stop if $P(\bar{w})-D(\bar{\alpha}) \leq \epsilon$ and output $\bar{w}, \bar{\alpha}$, and $P(\bar{w})-D(\bar{\alpha})$

Figure 1: The Generic Proximal Stochastic Dual Coordinate Ascent Algorithm 
The above theorem tells us that the runtime required to find an $\epsilon$ accurate solution, with probability of at least $1-\delta$, is

$$
O\left(d\left(n+\frac{R^{2}}{\lambda \gamma}\right) \cdot \log \left(\frac{D\left(\alpha^{*}\right)-D\left(\alpha^{(0)}\right)}{\epsilon}\right) \cdot \log \left(\frac{1}{\delta}\right)\right) .
$$

This yields the following corollary.

Corollary 1. The expected runtime required to minimize $P$ up to accuracy $\epsilon$ is

$$
O\left(d\left(n+\frac{R^{2}}{\lambda \gamma}\right) \cdot \log \left(\frac{D\left(\alpha^{*}\right)-D\left(\alpha^{(0)}\right)}{\epsilon}\right)\right)
$$

Proof. We have shown that with a runtime of $O\left(d\left(n+\frac{R^{2}}{\lambda \gamma}\right) \cdot \log \left(\frac{2\left(D\left(\alpha^{*}\right)-D\left(\alpha^{(0)}\right)\right)}{\epsilon}\right)\right)$ we can find an $\epsilon$ accurate solution with probability of at least $1 / 2$. Therefore, we can run the procedure for this amount of time and check if the duality gap is smaller than $\epsilon$. If yes, we are done. Otherwise, we would restart the process. Since the probability of success is $1 / 2$ we have that the average number of restarts we need is 2 , which concludes the proof.

\subsection{Acceleration}

The Prox-SDCA procedure described in the previous subsection has the iteration bound of $\tilde{O}\left(n+\frac{R^{2}}{\lambda \gamma}\right)$. This is a nearly linear runtime whenever the condition number, $R^{2} /(\lambda \gamma)$, is $O(n)$. In this section we show how to improve the dependence on the condition number by an acceleration procedure. In particular, throughout this section we assume that $10 n<\frac{R^{2}}{\lambda \gamma}$. We further assume throughout this subsection that the regularizer, $g$, is 1 -strongly convex with respect to the Euclidean norm, i.e. $\|u\|_{P^{\prime}}=\|\cdot\|_{2}$. This also implies that $\|u\|_{D^{\prime}}$ is the Euclidean norm. A generalization of the acceleration technique for strongly convex regularizers with respect to general norms is left to future work.

The main idea of the acceleration procedure is to iteratively run the Prox-SDCA procedure, where at iteration $t$ we call Prox-SDCA with the modified objective, $\tilde{P}_{t}(w)=P(w)+\frac{\kappa}{2}\left\|w-y^{(t-1)}\right\|^{2}$, where $\kappa$ is a relatively large regularization parameter and the regularization is centered around the vector

$$
y^{(t-1)}=w^{(t-1)}+\beta\left(w^{(t-1)}-w^{(t-2)}\right)
$$

for some $\beta \in(0,1)$. That is, our regularization is centered around the previous solution plus a "momentum term" $\beta\left(w^{(t-1)}-w^{(t-2)}\right)$.

A pseudo-code of the algorithm is given in Figure 2. Note that all the parameters of the algorithm are determined by our theory.

Remark 1. In the pseudo-code below, we specify the parameters based on our theoretical derivation. In our experiments, we found out that this choice of parameters also work very well in practice. However, we also found out that the algorithm is not very sensitive to the choice of parameters. For example, we found out that running $5 n$ iterations of Prox-SDCA (that is, 5 epochs over the data), without checking the stopping condition, also works very well.

The main theorem is the following.

Theorem 3. Consider the accelerated Prox-SDCA algorithm given in Figure 2. 


\section{Procedure Accelerated Prox-SDCA}

Goal: Minimize $P(w)=\frac{1}{n} \sum_{i=1}^{n} \phi_{i}\left(X_{i}^{\top} w\right)+\lambda g(w)$

Input: Target accuracy $\epsilon$ (only used in the stopping condition)

Assumptions:

$\forall i, \phi_{i}$ is $(1 / \gamma)$-smooth w.r.t. $\|\cdot\|_{P}$ and let $\|\cdot\|_{D}$ be the dual norm of $\|\cdot\|_{P}$

$g$ is 1-strongly convex w.r.t. $\|\cdot\|_{2}$

$\forall i,\left\|X_{i}\right\|_{D \rightarrow 2} \leq R$

$\frac{R^{2}}{\gamma \lambda}>10 n$ (otherwise, solve the problem using vanilla Prox-SDCA)

Define $\kappa=\frac{R^{2}}{\gamma n}-\lambda, \mu=\lambda / 2, \rho=\mu+\kappa, \eta=\sqrt{\mu / \rho}, \beta=\frac{1-\eta}{1+\eta}$,

Initialize $y^{(1)}=w^{(1)}=0, \alpha^{(1)}=0, \xi_{1}=\left(1+\eta^{-2}\right)(P(0)-D(0))$

Iterate: for $t=2,3, \ldots$

Let $\tilde{P}_{t}(w)=\frac{1}{n} \sum_{i=1}^{n} \phi_{i}\left(X_{i}^{\top} w\right)+\tilde{\lambda} \tilde{g}_{t}(w)$

where $\tilde{\lambda} \tilde{g}_{t}(w)=\lambda g(w)+\frac{\kappa}{2}\|w\|_{2}^{2}-\kappa w^{\top} y^{(t-1)}$

Call $\left(w^{(t)}, \alpha^{(t)}, \epsilon_{t}\right)=\operatorname{Prox}-\operatorname{SDCA}\left(\tilde{P}_{t}, \frac{\eta}{2\left(1+\eta^{-2}\right)} \xi_{t-1}, \alpha^{(t-1)}\right)$

Let $y^{(t)}=w^{(t)}+\beta\left(w^{(t)}-w^{(t-1)}\right)$

Let $\xi_{t}=(1-\eta / 2)^{t-1} \xi_{1}$

Stopping conditions: break and return $w^{(t)}$ if one of the following conditions hold:

1. $t \geq 1+\frac{2}{\eta} \log \left(\xi_{1} / \epsilon\right)$

2. $(1+\rho / \mu) \epsilon_{t}+\frac{\rho \kappa}{2 \mu}\left\|w^{(t)}-y^{(t-1)}\right\|^{2} \leq \epsilon$

Figure 2: The Accelerated Prox-SDCA Algorithm 
- Correctness: When the algorithm terminates we have that $P\left(w^{(t)}\right)-P\left(w^{*}\right) \leq \epsilon$.

- Runtime:

- The number of outer iterations is at most

$$
1+\frac{2}{\eta} \log \left(\xi_{1} / \epsilon\right) \leq 1+\sqrt{\frac{8 R^{2}}{\lambda \gamma n}}\left(\log \left(\frac{2 R^{2}}{\lambda \gamma n}\right)+\log \left(\frac{P(0)-D(0)}{\epsilon}\right)\right) .
$$

- Each outer iteration involves a single call to Prox-SDCA, and the averaged runtime required by each such call is

$$
O\left(d n \log \left(\frac{R^{2}}{\lambda \gamma n}\right)\right) \text {. }
$$

By a straightforward amplification argument we obtain that for every $\delta \in(0,1)$ the total runtime required by accelerated Prox-SDCA to guarantee an $\epsilon$-accurate solution with probability of at least $1-\delta$ is

$$
O\left(d \sqrt{\frac{n R^{2}}{\lambda \gamma}} \log \left(\frac{R^{2}}{\lambda \gamma n}\right)\left(\log \left(\frac{R^{2}}{\lambda \gamma n}\right)+\log \left(\frac{P(0)-D(0)}{\epsilon}\right)\right) \log \left(\frac{1}{\delta}\right)\right) .
$$

\subsection{Non-smooth, Lipschitz, loss functions}

So far we have assumed that for every $i, \phi_{i}$ is a $(1 / \gamma)$-smooth function. We now consider the case in which $\phi_{i}$ might be non-smooth, and even non-differentiable, but it is $L$-Lipschitz.

Following Nesterov [17], we apply a "smoothing" technique. We first observe that if $\phi$ is $L$-Lipschitz function then the domain of $\phi^{*}$ is in the ball of radius $L$.

Lemma 1. Let $\phi: \mathbb{R}^{k} \rightarrow \mathbb{R}$ be an L-Lipschitz function w.r.t. a norm $\|\cdot\|_{P}$ and let $\|\cdot\|_{D}$ be the dual norm. Then, for any $\alpha \in \mathbb{R}^{k}$ s.t. $\|\alpha\|_{D}>L$ we have that $\phi^{*}(\alpha)=\infty$.

Proof. Fix some $\alpha$ with $\|\alpha\|_{D}>L$. Let $x_{0}$ be a vector such that $\left\|x_{0}\right\|_{P}=1$ and $\alpha^{\top} x_{0}=\|\alpha\|_{D}$ (this is a vector that achieves the maximal objective in the definition of the dual norm). By definition of the conjugate we have

$$
\begin{aligned}
\phi^{*}(\alpha) & =\sup _{x}\left[\alpha^{\top} x-\phi(x)\right] \\
& =-\phi(0)+\sup _{x}\left[\alpha^{\top} x-(\phi(x)-\phi(0))\right] \\
& \geq-\phi(0)+\sup _{x}\left[\alpha^{\top} x-L\|x-0\|_{P}\right] \\
& \geq-\phi(0)+\sup _{c>0}\left[\alpha^{\top}\left(c x_{0}\right)-L\left\|c x_{0}\right\|_{P}\right] \\
& =-\phi(0)+\sup _{c>0}\left(\|\alpha\|_{D}-L\right) c=\infty
\end{aligned}
$$

This observation allows us to smooth $L$-Lipschitz functions by adding regularization to their conjugate. In particular, the following lemma generalizes Lemma 2.5 in [26]. 
Lemma 2. Let $\phi$ be a proper, convex, L-Lipschitz function w.r.t. a norm $\|\cdot\|_{P}$, let $\|\cdot\|_{D}$ be the dual norm, and let $\phi^{*}$ be the conjugate of $\phi$. Assume that $\|\cdot\|_{2} \leq\|\cdot\|_{D}$. Define $\tilde{\phi}^{*}(\alpha)=\phi^{*}(\alpha)+\frac{\gamma}{2}\|\alpha\|_{2}^{2}$ and let $\tilde{\phi}$ be the conjugate of $\tilde{\phi}^{*}$. Then, $\tilde{\phi}$ is $(1 / \gamma)$-smooth w.r.t. the Euclidean norm and

$$
\forall a, \quad 0 \leq \phi(a)-\tilde{\phi}(a) \leq \gamma L^{2} / 2 .
$$

Proof. The fact that $\tilde{\phi}$ is $(1 / \gamma)$-smooth follows directly from the fact that $\tilde{\phi}^{*}$ is $\gamma$-strongly convex. For the second claim note that

$$
\tilde{\phi}(a)=\sup _{b}\left[b a-\phi^{*}(b)-\frac{\gamma}{2}\|b\|_{2}^{2}\right] \leq \sup _{b}\left[b a-\phi^{*}(b)\right]=\phi(a)
$$

and

$$
\begin{aligned}
\tilde{\phi}(a) & =\sup _{b}\left[b a-\phi^{*}(b)-\frac{\gamma}{2}\|b\|_{2}^{2}\right]=\sup _{b:\|b\|_{D} \leq L}\left[b a-\phi^{*}(b)-\frac{\gamma}{2}\|b\|_{2}^{2}\right] \\
& \geq \sup _{b:\|b\|_{D} \leq L}\left[b a-\phi^{*}(b)-\frac{\gamma}{2}\|b\|_{D}^{2}\right] \geq \sup _{b:\|b\|_{D} \leq L}\left[b a-\phi^{*}(b)\right]-\frac{\gamma}{2} L^{2} \\
& =\phi(a)-\frac{\gamma}{2} L^{2} .
\end{aligned}
$$

Remark 2. It is also possible to smooth using different regularization functions which are strongly convex with respect to other norms. See Nesterov [17] for discussion.

\section{Proof of Theorem 3}

The first claim of the theorem is that when the procedure stops we have $P\left(w^{(t)}\right)-P\left(w^{*}\right) \leq \epsilon$. We therefore need to show that each stopping condition guarantees that $P\left(w^{(t)}\right)-P\left(w^{*}\right) \leq \epsilon$.

For the second stopping condition, recall that $w^{(t)}$ is an $\epsilon_{t}$-accurate minimizer of $P(w)+\frac{\kappa}{2}\left\|w-y^{(t-1)}\right\|^{2}$, and hence by Lemma 3 below (with $z=w^{*}, w^{+}=w^{(t)}$, and $y=y^{(t-1)}$ ):

$$
\begin{aligned}
P\left(w^{*}\right) & \geq P\left(w^{(t)}\right)+Q_{\epsilon}\left(w^{*} ; w^{(t)}, y^{(t-1)}\right) \\
& \geq P\left(w^{(t)}\right)-\frac{\rho \kappa}{2 \mu}\left\|y^{(t-1)}-w^{(t)}\right\|^{2}-(1+\rho / \mu) \epsilon_{t} .
\end{aligned}
$$

It is left to show that the first stopping condition is correct, namely, to show that after $1+\frac{2}{\eta} \log \left(\xi_{1} / \epsilon\right)$ iterations the algorithm must converge to an $\epsilon$-accurate solution. Observe that the definition of $\xi_{t}$ yields that $\xi_{t}=(1-\eta / 2)^{t-1} \xi_{1} \leq e^{-\eta(t-1) / 2} \xi_{1}$. Therefore, to prove that the first stopping condition is valid, it suffices to show that for every $t, P\left(w^{(t)}\right)-P\left(w^{*}\right) \leq \xi_{t}$.

Recall that at each outer iteration of the accelerated procedure, we approximately minimize an objective of the form

$$
P(w ; y)=P(w)+\frac{\kappa}{2}\|w-y\|^{2} .
$$

Of course, minimizing $P(w ; y)$ is not the same as minimizing $P(w)$. Our first lemma shows that for every $y$, if $w^{+}$is an $\epsilon$-accurate minimizer of $P(w ; y)$ then we can derive a lower bound on $P(w)$ based on $P\left(w^{+}\right)$ and a convex quadratic function of $w$. 
Lemma 3. Let $\mu=\lambda / 2$ and $\rho=\mu+\kappa$. Let $w^{+}$be a vector such that $P\left(w^{+} ; y\right) \leq \min _{w} P(w, y)+\epsilon$. Then, for every $z$,

$$
P(z) \geq P\left(w^{+}\right)+Q_{\epsilon}\left(z ; w^{+}, y\right)
$$

where

$$
Q_{\epsilon}\left(z ; w^{+}, y\right)=\frac{\mu}{2}\left\|z-\left(y-\frac{\rho}{\mu}\left(y-w^{+}\right)\right)\right\|^{2}-\frac{\rho \kappa}{2 \mu}\left\|y-w^{+}\right\|^{2}-(1+\rho / \mu) \epsilon .
$$

Proof. Denote

$$
\Psi(w)=P(w)-\frac{\mu}{2}\|w\|^{2}
$$

We can write

$$
\frac{1}{2}\|w\|^{2}=\frac{1}{2}\|y\|^{2}+y^{\top}(w-y)+\frac{1}{2}\|w-y\|^{2} .
$$

It follows that

$$
P(w)=\Psi(w)+\frac{\mu}{2}\|w\|^{2}=\Psi(w)+\frac{\mu}{2}\|y\|^{2}+\mu y^{\top}(w-y)+\frac{\mu}{2}\|w-y\|^{2} .
$$

Therefore, we can rewrite $P(w ; y)$ as:

$$
P(w ; y)=\Psi(w)+\frac{\mu}{2}\|y\|^{2}+\mu y^{\top}(w-y)+\frac{\rho}{2}\|w-y\|^{2} .
$$

Let $\tilde{w}=\operatorname{argmin}_{w} P(w ; y)$. Therefore, the gradien $\bigsqcup^{2}$ of $P(w ; y)$ w.r.t. $w$ vanishes at $\tilde{w}$, which yields

$$
\nabla \Psi(\tilde{w})+\mu y+\rho(\tilde{w}-y)=0 \Rightarrow \nabla \Psi(\tilde{w})+\mu y=\rho(y-\tilde{w}) .
$$

By the $\mu$-strong convexity of $\Psi$ we have that for every $z$,

$$
\Psi(z) \geq \Psi(\tilde{w})+\nabla \Psi(\tilde{w})^{\top}(z-\tilde{w})+\frac{\mu}{2}\|z-\tilde{w}\|^{2} .
$$

Therefore,

$$
\begin{aligned}
P(z) & =\Psi(z)+\frac{\mu}{2}\|y\|^{2}+\mu y^{\top}(z-y)+\frac{\mu}{2}\|z-y\|^{2} \\
& \geq \Psi(\tilde{w})+\nabla \Psi(\tilde{w})^{\top}(z-\tilde{w})+\frac{\mu}{2}\|z-\tilde{w}\|^{2}+\frac{\mu}{2}\|y\|^{2}+\mu y^{\top}(z-y)+\frac{\mu}{2}\|z-y\|^{2} \\
& =P(\tilde{w} ; y)-\frac{\rho}{2}\|\tilde{w}-y\|^{2}+\nabla \Psi(\tilde{w})^{\top}(z-\tilde{w})+\mu y^{\top}(z-\tilde{w})+\frac{\mu}{2}\left(\|z-\tilde{w}\|^{2}+\|z-y\|^{2}\right) \\
& =P(\tilde{w} ; y)-\frac{\rho}{2}\|\tilde{w}-y\|^{2}+\rho(y-\tilde{w})^{\top}(z-\tilde{w})+\frac{\mu}{2}\left(\|z-\tilde{w}\|^{2}+\|z-y\|^{2}\right) \\
& =P(\tilde{w} ; y)+\frac{\rho}{2}\|\tilde{w}-y\|^{2}+\rho(y-\tilde{w})^{\top}(z-y)+\frac{\mu}{2}\left(\|z-\tilde{w}\|^{2}+\|z-y\|^{2}\right) .
\end{aligned}
$$

\footnotetext{
${ }^{2}$ If the regularizer $g(w)$ in the definition of $P(w)$ is non-differentiable, we can replace $\nabla \Psi(\tilde{w})$ with an appropriate sub-gradient of $\Psi$ at $\tilde{w}$. It is easy to verify that the proof is still valid.
} 
In addition, by standard algebraic manipulations,

$$
\begin{aligned}
& \frac{\rho}{2}\|\tilde{w}-y\|^{2}+\rho(y-\tilde{w})^{\top}(z-y)+\frac{\mu}{2}\|z-\tilde{w}\|^{2}-\left(\frac{\rho}{2}\left\|w^{+}-y\right\|^{2}+\rho\left(y-w^{+}\right)^{\top}(z-y)+\frac{\mu}{2}\left\|z-w^{+}\right\|^{2}\right) \\
& =\left(\rho\left(w^{+}-y\right)-\rho(z-y)+\mu\left(w^{+}-z\right)\right)^{\top}\left(\tilde{w}-w^{+}\right)+\frac{\rho+\mu}{2}\left\|\tilde{w}-w^{+}\right\|^{2} \\
& =(\rho+\mu)\left(w^{+}-z\right)^{\top}\left(\tilde{w}-w^{+}\right)+\frac{\rho+\mu}{2}\left\|\tilde{w}-w^{+}\right\|^{2} \\
& =\frac{1}{2}\left\|\sqrt{\mu}\left(w^{+}-z\right)+\frac{\rho+\mu}{\sqrt{\mu}}\left(\tilde{w}-w^{+}\right)\right\|^{2}-\frac{\mu}{2}\left\|z-w^{+}\right\|^{2}-\frac{(\rho+\mu)^{2}}{2 \mu}\left\|\tilde{w}-w^{+}\right\|^{2}+\frac{\rho+\mu}{2}\left\|\tilde{w}-w^{+}\right\|^{2} \\
& \geq-\frac{\mu}{2}\left\|z-w^{+}\right\|^{2}-\frac{\rho(\rho+\mu)}{2 \mu}\left\|\tilde{w}-w^{+}\right\|^{2} .
\end{aligned}
$$

Since $P(\cdot ; y)$ is $(\rho+\mu)$-strongly convex and $\tilde{w}$ minimizes $P(\cdot ; y)$, we have that for every $w^{+}$it holds that $\frac{\rho+\mu}{2}\left\|\tilde{w}-w^{+}\right\|^{2} \leq P\left(w^{+} ; y\right)-P(\tilde{w} ; y)$. Combining all the above and using the fact that for every $w, y$, $P(w ; y) \geq P(w)$, we obtain that for every $w^{+}$,

$P(z) \geq P\left(w^{+}\right)+\frac{\rho}{2}\left\|w^{+}-y\right\|^{2}+\rho\left(y-w^{+}\right)^{\top}(z-y)+\frac{\mu}{2}\|z-y\|^{2}-\left(1+\frac{\rho}{\mu}\right)\left(P\left(w^{+} ; y\right)-P(\tilde{w} ; y)\right)$.

Finally, using the assumption $P\left(w^{+} ; y\right) \leq \min _{w} P(w ; y)+\epsilon$ we conclude our proof.

We saw that the quadratic function $P\left(w^{+}\right)+Q_{\epsilon}\left(z ; w^{+}, y\right)$ lower bounds the function $P$ everywhere. Therefore, any convex combination of such functions would form a quadratic function which lower bounds $P$. In particular, the algorithm (implicitly) maintains a sequence of quadratic functions, $h_{1}, h_{2}, \ldots$, defined as follows. Choose $\eta \in(0,1)$ and a sequence $y^{(1)}, y^{(2)}, \ldots$ that will be specified later. Define,

$$
h_{1}(z)=P(0)+Q_{P(0)-D(0)}(z ; 0,0)=P(0)+\frac{\mu}{2}\|z\|^{2}-(1+\rho / \mu)(P(0)-D(0)),
$$

and for $t \geq 1$,

$$
h_{t+1}(z)=(1-\eta) h_{t}(z)+\eta\left(P\left(w^{(t+1)}\right)+Q_{\epsilon t+1}\left(z ; w^{(t+1)}, y^{(t)}\right)\right) .
$$

The following simple lemma shows that for every $t \geq 1$ and $z, h_{t}(z)$ lower bounds $P(z)$.

Lemma 4. Let $\eta \in(0,1)$ and let $y^{(1)}, y^{(2)}, \ldots$ be any sequence of vectors. Assume that $w^{(1)}=0$ and for every $t \geq 1, w^{(t+1)}$ satisfies $P\left(w^{(t+1)} ; y^{(t)}\right) \leq \min _{w} P\left(w ; y^{(t)}\right)+\epsilon_{t+1}$. Then, for every $t \geq 1$ and every vector $z$ we have

$$
h_{t}(z) \leq P(z)
$$

Proof. The proof is by induction. For $t=1$, observe that $P(0 ; 0)=P(0)$ and that for every $w$ we have $P(w ; 0) \geq P(w) \geq D(0)$. This yields $P(0 ; 0)-\min _{w} P(w ; 0) \leq P(0)-D(0)$. The claim now follows directly from Lemma 3. Next, for the inductive step, assume the claim holds for some $t-1 \geq 1$ and let us prove it for $t$. By the recursive definition of $h_{t}$ and by using Lemma 3 we have

$$
h_{t}(z)=(1-\eta) h_{t-1}(z)+\eta\left(P\left(w^{(t)}\right)+Q_{\epsilon_{t}}\left(z ; w^{(t)}, y^{(t-1)}\right)\right) \leq(1-\eta) h_{t-1}(z)+\eta P(z) .
$$

Using the inductive assumption we obtain that the right-hand side of the above is upper bounded by ( 1 п) $P(z)+\eta P(z)=P(z)$, which concludes our proof. 
The more difficult part of the proof is to show that for every $t \geq 1$,

$$
P\left(w^{(t)}\right) \leq \min _{w} h_{t}(w)+\xi_{t}
$$

If this holds true, then we would immediately get that for every $w^{*}$,

$$
P\left(w^{(t)}\right)-P\left(w^{*}\right) \leq P\left(w^{(t)}\right)-h_{t}\left(w^{*}\right) \leq P\left(w^{(t)}\right)-\min _{w} h_{t}(w) \leq \xi_{t} .
$$

This will conclude the proof of the first part of Theorem 3 , since $\xi_{t}=\xi_{1}(1-\eta / 2)^{t-1} \leq \xi_{1} e^{-(t-1) \eta / 2}$, and therefore, $1+\frac{2}{\eta} \log \left(\xi_{1} / \epsilon\right)$ iterations suffice to guarantee that $P\left(w^{(t)}\right)-P\left(w^{*}\right) \leq \epsilon$.

Define

$$
v^{(t)}=\underset{w}{\operatorname{argmin}} h_{t}(w) .
$$

Let us construct an explicit formula for $v^{(t)}$. Clearly, $v^{(1)}=0$. Assume that we have calculated $v^{(t)}$ and let us calculate $v^{(t+1)}$. Note that $h_{t}$ is a quadratic function which is minimized at $v^{(t)}$. Furthermore, it is easy to see that for every $t, h_{t}$ is $\mu$-strongly convex quadratic function. Therefore,

$$
h_{t}(z)=h_{t}\left(v^{(t)}\right)+\frac{\mu}{2}\left\|z-v^{(t)}\right\|^{2} .
$$

By the definition of $h_{t+1}$ we obtain that

$$
h_{t+1}(z)=(1-\eta)\left(h_{t}\left(v^{(t)}\right)+\frac{\mu}{2}\left\|z-v^{(t)}\right\|^{2}\right)+\eta\left(P\left(w^{(t+1)}\right)+Q_{\epsilon t+1}\left(z ; w^{(t+1)}, y^{(t)}\right)\right) .
$$

Since the gradient of $h_{t+1}(z)$ at $v^{(t+1)}$ should be zero, we obtain that $v^{(t+1)}$ should satisfy

$$
(1-\eta) \mu\left(v^{(t+1)}-v^{(t)}\right)+\eta \mu\left(v^{(t+1)}-\left(y^{(t)}-\frac{\rho}{\mu}\left(y^{(t)}-w^{(t+1)}\right)\right)\right)=0
$$

Rearranging, we obtain

$$
v^{(t+1)}=(1-\eta) v^{(t)}+\eta\left(y^{(t)}-\frac{\rho}{\mu}\left(y^{(t)}-w^{(t+1)}\right)\right) .
$$

Getting back to our second phase of the proof, we need to show that for every $t$ we have $P\left(w^{(t)}\right) \leq$ $h_{t}\left(v^{(t)}\right)+\xi_{t}$. We do so by induction. For the case $t=1$ we have

$$
P\left(w^{(1)}\right)-h_{1}\left(v^{(1)}\right)=P(0)-h_{1}(0)=(1+\rho / \mu)(P(0)-D(0))=\xi_{1} .
$$

For the induction step, assume the claim holds for $t \geq 1$ and let us prove it for $t+1$. We use the shorthands,

$$
Q_{t}(z)=Q_{\epsilon_{t}}\left(z ; w^{(t)}, y^{(t-1)}\right) \text { and } \psi_{t}(z)=Q_{t}(z)+P\left(w^{(t)}\right) .
$$

Let us rewrite $h_{t+1}\left(v^{(t+1)}\right)$ as

$$
\begin{aligned}
h_{t+1}\left(v^{(t+1)}\right) & =(1-\eta) h_{t}\left(v^{(t+1)}\right)+\eta \psi_{t+1}\left(v^{(t+1)}\right) \\
& =(1-\eta)\left(h_{t}\left(v^{(t)}\right)+\frac{\mu}{2}\left\|v^{(t)}-v^{(t+1)}\right\|^{2}\right)+\eta \psi_{t+1}\left(v^{(t+1)}\right) .
\end{aligned}
$$


By the inductive assumption we have $h_{t}\left(v^{(t)}\right) \geq P\left(w^{(t)}\right)-\xi_{t}$ and by Lemma 3 we have $P\left(w^{(t)}\right) \geq$ $\psi_{t+1}\left(w^{(t)}\right)$. Therefore,

$$
\begin{aligned}
h_{t+1}\left(v^{(t+1)}\right) & \geq(1-\eta)\left(\psi_{t+1}\left(w^{(t)}\right)-\xi_{t}+\frac{\mu}{2}\left\|v^{(t)}-v^{(t+1)}\right\|^{2}\right)+\eta \psi_{t+1}\left(v^{(t+1)}\right) \\
& =\frac{(1-\eta) \mu}{2}\left\|v^{(t)}-v^{(t+1)}\right\|^{2}+\eta \psi_{t+1}\left(v^{(t+1)}\right)+(1-\eta) \psi_{t+1}\left(w^{(t)}\right)-(1-\eta) \xi_{t}
\end{aligned}
$$

Next, note that we can rewrite

$$
Q_{t+1}(z)=\frac{\mu}{2}\left\|z-y^{(t)}\right\|^{2}+\rho\left(z-y^{(t)}\right)^{\top}\left(y^{(t)}-w^{(t+1)}\right)+\frac{\rho}{2}\left\|y^{(t)}-w^{(t+1)}\right\|^{2}-(1+\rho / \mu) \epsilon_{t+1} .
$$

Therefore,

$$
\begin{aligned}
& \eta \psi_{t+1}\left(v^{(t+1)}\right)+(1-\eta) \psi_{t+1}\left(w^{(t)}\right)-P\left(w^{(t+1)}\right)+(1+\rho / \mu) \epsilon_{t+1} \\
& =\frac{\eta \mu}{2}\left\|v^{(t+1)}-y^{(t)}\right\|^{2}+\frac{(1-\eta) \mu}{2}\left\|w^{(t)}-y^{(t)}\right\|^{2}+\rho\left(\eta v^{(t+1)}+(1-\eta) w^{(t)}-y^{(t)}\right)^{\top}\left(y^{(t)}-w^{(t+1)}\right) \\
& +\frac{\rho}{2}\left\|y^{(t)}-w^{(t+1)}\right\|^{2}
\end{aligned}
$$

So far we did not specify $\eta$ and $y^{(t)}\left(\operatorname{except} y^{(0)}=0\right)$. We next set

$$
\eta=\sqrt{\mu / \rho} \text { and } \forall t \geq 1, y^{(t)}=(1+\eta)^{-1}\left(\eta v^{(t)}+w^{(t)}\right) .
$$

This choices guarantees that (see [5])

$$
\begin{aligned}
\eta v^{(t+1)}+(1-\eta) w^{(t)} & =\eta(1-\eta) v^{(t)}+\eta^{2}\left(1-\frac{\rho}{\mu}\right) y^{(t)}+\eta^{2} \frac{\rho}{\mu} w^{(t+1)}+(1-\eta) w^{(t)} \\
& =w^{(t+1)}+(1-\eta)\left[\eta v^{(t)}+\frac{\eta^{2}\left(1-\frac{\rho}{\mu}\right)}{1-\eta} y^{(t)}+w^{(t)}\right] \\
& =w^{(t+1)}+(1-\eta)\left[\eta v^{(t)}-\frac{1-\eta^{2}}{1-\eta} y^{(t)}+w^{(t)}\right] \\
& =w^{(t+1)}+(1-\eta)\left[\eta v^{(t)}-(1+\eta) y^{(t)}+w^{(t)}\right] \\
& =w^{(t+1)}
\end{aligned}
$$

We also observe that $\epsilon_{t+1} \leq \frac{\eta \xi_{t}}{2\left(1+\eta^{-2}\right)}$ which implies that $(1+\rho / \mu) \epsilon_{t+1}+(1-\eta) \xi_{t} \leq(1-\eta / 2) \xi_{t}=\xi_{t+1}$. Combining the above with (6) and (7), and rearranging terms, we obtain that

$$
\begin{aligned}
& h_{t+1}\left(v^{(t+1)}\right)-P\left(w^{(t+1)}\right)+\xi_{t+1}-\frac{(1-\eta) \mu}{2}\left\|w^{(t)}-y^{(t)}\right\|^{2} \\
& \geq \frac{(1-\eta) \mu}{2}\left\|v^{(t)}-v^{(t+1)}\right\|^{2}+\frac{\eta \mu}{2}\left\|v^{(t+1)}-y^{(t)}\right\|^{2}-\frac{\rho}{2}\left\|y^{(t)}-w^{(t+1)}\right\|^{2} .
\end{aligned}
$$

Next, observe that $\rho \eta^{2}=\mu$ and that by (5) we have

$$
y^{(t)}-w^{(t+1)}=\eta\left[\eta y^{(t)}+(1-\eta) v^{(t)}-v^{(t+1)}\right] .
$$


We therefore obtain that

$$
\begin{aligned}
& h_{t+1}\left(v^{(t+1)}\right)-P\left(w^{(t+1)}\right)+\xi_{t+1}-\frac{(1-\eta) \mu}{2}\left\|w^{(t)}-y^{(t)}\right\|^{2} \\
& \geq \frac{(1-\eta) \mu}{2}\left\|v^{(t)}-v^{(t+1)}\right\|^{2}+\frac{\eta \mu}{2}\left\|y^{(t)}-v^{(t+1)}\right\|^{2}-\frac{\mu}{2}\left\|\eta y^{(t)}+(1-\eta) v^{(t)}-v^{(t+1)}\right\|^{2} .
\end{aligned}
$$

The right-hand side of the above is non-negative because of the convexity of the function $f(z)=\frac{\mu}{2} \| z-$ $v^{(t+1)} \|^{2}$, which yields

$$
P\left(w^{(t+1)}\right) \leq h_{t+1}\left(v^{(t+1)}\right)+\xi_{t+1}-\frac{(1-\eta) \mu}{2}\left\|w^{(t)}-y^{(t)}\right\|^{2} \leq h_{t+1}\left(v^{(t+1)}\right)+\xi_{t+1} .
$$

This concludes our inductive argument.

Proving the "runtime" part of Theorem 3; We next show that each call to Prox-SDCA will terminate quickly. By the definition of $\kappa$ we have that

$$
\frac{R^{2}}{(\kappa+\lambda) \gamma}=n
$$

Therefore, based on Corollary 1 we know that the averaged runtime at iteration $t$ is

$$
O\left(d n \log \left(\frac{\tilde{D}_{t}\left(\alpha^{*}\right)-\tilde{D}_{t}\left(\alpha^{(t-1)}\right)}{\frac{\eta}{2\left(1+\eta^{-2}\right)} \xi_{t-1}}\right)\right) .
$$

The following lemma bounds the initial dual sub-optimality at iteration $t \geq 4$. Similar arguments will yield a similar result for $t<4$.

\section{Lemma 5.}

$$
\tilde{D}_{t}\left(\alpha^{*}\right)-\tilde{D}_{t}\left(\alpha^{(t-1)}\right) \leq \epsilon_{t-1}+\frac{36 \kappa}{\lambda} \xi_{t-3}
$$

Proof. Define $\tilde{\lambda}=\lambda+\kappa, f(w)=\frac{\lambda}{\tilde{\lambda}} g(w)+\frac{\kappa}{2 \tilde{\lambda}}\|w\|^{2}$, and $\tilde{g}_{t}(w)=f(w)-\frac{\kappa}{\tilde{\lambda}} w^{\top} y^{(t-1)}$. Note that $\tilde{\lambda}$ does not depend on $t$ and therefore $v(\alpha)=\frac{1}{n \tilde{\lambda}} \sum_{i} X_{i} \alpha_{i}$ is the same for every $t$. Let,

$$
\tilde{P}_{t}(w)=\frac{1}{n} \sum_{i=1}^{n} \phi_{i}\left(X_{i}^{\top} w\right)+\tilde{\lambda} \tilde{g}_{t}(w) .
$$

We have

$$
\tilde{P}_{t}\left(w^{(t-1)}\right)=\tilde{P}_{t-1}\left(w^{(t-1)}\right)+\kappa w^{(t-1) \top}\left(y^{(t-2)}-y^{(t-1)}\right) .
$$

Since

$$
\tilde{g}_{t}^{*}(\theta)=\max _{w} w^{\top}\left(\theta+\frac{\kappa}{\tilde{\lambda}} y^{(t-1)}\right)-f(w)=f^{*}\left(\theta+\frac{\kappa}{\tilde{\lambda}} y^{(t-1)}\right),
$$

we obtain that the dual problem is

$$
\tilde{D}_{t}(\alpha)=-\frac{1}{n} \sum_{i} \phi_{i}^{*}\left(-\alpha_{i}\right)-\tilde{\lambda} f^{*}\left(v(\alpha)+\frac{\kappa}{\tilde{\lambda}} y^{(t-1)}\right)
$$


Let $z=\frac{\kappa}{\tilde{\lambda}}\left(y^{(t-1)}-y^{(t-2)}\right)$, then, by the smoothness of $f^{*}$ we have $f^{*}\left(v(\alpha)+\frac{\kappa}{\tilde{\lambda}} y^{(t-1)}\right)=f^{*}\left(v(\alpha)+\frac{\kappa}{\tilde{\lambda}} y^{(t-2)}+z\right) \leq f^{*}\left(v(\alpha)+\frac{\kappa}{\tilde{\lambda}} y^{(t-2)}\right)+\nabla f^{*}\left(v(\alpha)+\frac{\kappa}{\tilde{\lambda}} y^{(t-2)}\right)^{\top} z+\frac{1}{2}\|z\|^{2}$.

Applying this for $\alpha^{(t-1)}$ and using $w^{(t-1)}=\nabla \tilde{g}_{t-1}^{*}\left(v\left(\alpha^{(t-1)}\right)\right)=\nabla f^{*}\left(v\left(\alpha^{(t-1)}\right)+\frac{\kappa}{\tilde{\lambda}} y^{(t-2)}\right)$, we obtain

$$
f^{*}\left(v\left(\alpha^{(t-1)}\right)+\frac{\kappa}{\tilde{\lambda}} y^{(t-1)}\right) \leq f^{*}\left(v\left(\alpha^{(t-1)}\right)+\frac{\kappa}{\tilde{\lambda}} y^{(t-2)}\right)+w^{(t-1) \top} z+\frac{1}{2}\|z\|^{2} .
$$

It follows that

$$
-\tilde{D}_{t}\left(\alpha^{(t-1)}\right)+\tilde{D}_{t-1}\left(\alpha^{(t-1)}\right) \leq \kappa w^{(t-1) \top}\left(y^{(t-1)}-y^{(t-2)}\right)+\frac{\kappa^{2}}{2 \tilde{\lambda}}\left\|y^{(t-1)}-y^{(t-2)}\right\|^{2} .
$$

Combining the above with (8), we obtain that

$$
\tilde{P}_{t}\left(w^{(t-1)}\right)-\tilde{D}_{t}\left(\alpha^{(t-1)}\right) \leq \tilde{P}_{t-1}\left(w^{(t-1)}\right)-\tilde{D}_{t-1}\left(\alpha^{(t-1)}\right)+\frac{\kappa^{2}}{2 \tilde{\lambda}}\left\|y^{(t-1)}-y^{(t-2)}\right\|^{2} .
$$

Since $\tilde{P}_{t}\left(w^{(t-1)}\right) \geq \tilde{D}_{t}\left(\alpha^{*}\right)$ and since $\tilde{\lambda} \geq \kappa$ we get that

$$
\tilde{D}_{t}\left(\alpha^{*}\right)-\tilde{D}_{t}\left(\alpha^{(t-1)}\right) \leq \epsilon_{t-1}+\frac{\kappa}{2}\left\|y^{(t-1)}-y^{(t-2)}\right\|^{2} .
$$

Next, we bound $\left\|y^{(t-1)}-y^{(t-2)}\right\|^{2}$. We have

$$
\begin{aligned}
\left\|y^{(t-1)}-y^{(t-2)}\right\| & =\left\|w^{(t-1)}-w^{(t-2)}+\beta\left(w^{(t-1)}-w^{(t-2)}-w^{(t-2)}+w^{(t-3)}\right)\right\| \\
& \leq 3 \max _{i \in\{1,2\}}\left\|w^{(t-i)}-w^{(t-i-1)}\right\|,
\end{aligned}
$$

where we used the triangle inequality and $\beta<1$. By strong convexity of $P$ we have, for every $i$,

$$
\left\|w^{(i)}-w^{*}\right\| \leq \sqrt{\frac{P\left(w^{(i)}\right)-P\left(w^{*}\right)}{\lambda / 2}} \leq \sqrt{\frac{\xi_{i}}{\lambda / 2}},
$$

which implies

$$
\left\|w^{(t-i)}-w^{(t-i-1)}\right\| \leq\left\|w^{(t-i)}-w^{*}\right\|+\left\|w^{*}-w^{(t-i-1)}\right\| \leq 2 \sqrt{\frac{\xi_{t-i-1}}{\lambda / 2}} .
$$

This yields the bound

$$
\left\|y^{(t-1)}-y^{(t-2)}\right\|^{2} \leq 72 \frac{\xi_{t-3}}{\lambda}
$$

All in all, we have obtained that

$$
\tilde{D}_{t}\left(\alpha^{*}\right)-\tilde{D}_{t}\left(\alpha^{(t-1)}\right) \leq \epsilon_{t-1}+\frac{36 \kappa}{\lambda} \xi_{t-3} .
$$


Getting back to the proof of the second claim of Theorem 3, we have obtained that

$$
\begin{aligned}
\frac{\tilde{D}_{t}\left(\alpha^{*}\right)-\tilde{D}_{t}\left(\alpha^{(t-1)}\right)}{\frac{\eta}{2\left(1+\eta^{-2}\right)} \xi_{t-1}} & \leq \frac{\epsilon_{t-1}}{\frac{\eta}{2\left(1+\eta^{-2}\right)} \xi_{t-1}}+\frac{36 \kappa \xi_{t-3}}{\lambda \frac{\eta}{2\left(1+\eta^{-2}\right)} \xi_{t-1}} \\
& \leq(1-\eta / 2)^{-1}+\frac{36 \kappa 2\left(1+\eta^{-2}\right)}{\lambda \eta}(1-\eta / 2)^{-2} \\
& \leq(1-\eta / 2)^{-4}\left(1+\frac{72 \kappa\left(1+\eta^{-2}\right)}{\lambda \eta}\right) \\
& \leq(1-\eta / 2)^{-2}\left(1+36 \eta^{-5}\right)
\end{aligned}
$$

where in the last inequality we used $\eta^{-2}-1=\frac{2 \kappa}{\lambda}$, which implies that $\frac{2 \kappa}{\lambda}\left(1+\eta^{-2}\right) \leq \eta^{-4}$. Using $1<\eta^{-5}$, $1-\eta / 2 \geq 0.5$, and taking $\log$ to both sides, we get that

$$
\log \left(\frac{\tilde{D}_{t}\left(\alpha^{*}\right)-\tilde{D}_{t}\left(\alpha^{(t-1)}\right)}{\frac{\eta}{2\left(1+\eta^{-2}\right)} \xi_{t-1}}\right) \leq 2 \log (2)+\log (37)-5 \log (\eta) \leq 7+2.5 \log \left(\frac{R^{2}}{\lambda \gamma n}\right) .
$$

All in all, we have shown that the average runtime required by $\operatorname{Prox}-\operatorname{SDCA}\left(\tilde{P}_{t}, \frac{\eta}{2\left(1+\eta^{-2}\right)} \xi_{t-1}, \alpha^{(t-1)}\right)$ is upper bounded by

$$
O\left(d n \log \left(\frac{R^{2}}{\lambda \gamma n}\right)\right)
$$

which concludes the proof of the second claim of Theorem 3 .

\section{Applications}

In this section we specify our algorithmic framework to several popular machine learning applications. In Section 5.1 we start by describing several loss functions and deriving their conjugate. In Section 5.2 we describe several regularization functions. Finally, in the rest of the subsections we specify our algorithm for Ridge regression, SVM, Lasso, logistic regression, and multiclass prediction.

\subsection{Loss functions}

Squared loss: $\quad \phi(a)=\frac{1}{2}(a-y)^{2}$ for some $y \in \mathbb{R}$. The conjugate function is

$$
\phi^{*}(b)=\max _{a} a b-\frac{1}{2}(a-y)^{2}=\frac{1}{2} b^{2}+y b
$$

Logistic loss: $\phi(a)=\log \left(1+e^{a}\right)$. The derivative is $\phi^{\prime}(a)=1 /\left(1+e^{-a}\right)$ and the second derivative is $\phi^{\prime \prime}(a)=\frac{1}{\left(1+e^{-a}\right)\left(1+e^{a}\right)} \in[0,1 / 4]$, from which it follows that $\phi$ is $(1 / 4)$-smooth. The conjugate function is

$$
\phi^{*}(b)=\max _{a} a b-\log \left(1+e^{a}\right)= \begin{cases}b \log (b)+(1-b) \log (1-b) & \text { if } b \in[0,1] \\ \infty & \text { otherwise }\end{cases}
$$

Hinge loss: $\quad \phi(a)=[1-a]_{+}:=\max \{0,1-a\}$. The conjugate function is

$$
\phi^{*}(b)=\max _{a} a b-\max \{0,1-a\}= \begin{cases}b & \text { if } b \in[-1,0] \\ \infty & \text { otherwise }\end{cases}
$$


Smooth hinge loss: This loss is obtained by smoothing the hinge-loss using the technique described in Lemma 2. This loss is parameterized by a scalar $\gamma>0$ and is defined as:

$$
\tilde{\phi}_{\gamma}(a)= \begin{cases}0 & a \geq 1 \\ 1-a-\gamma / 2 & a \leq 1-\gamma \\ \frac{1}{2 \gamma}(1-a)^{2} & \text { o.w. }\end{cases}
$$

The conjugate function is

$$
\tilde{\phi}_{\gamma}^{*}(b)= \begin{cases}b+\frac{\gamma}{2} b^{2} & \text { if } b \in[-1,0] \\ \infty & \text { otherwise }\end{cases}
$$

It follows that $\tilde{\phi}_{\gamma}^{*}$ is $\gamma$ strongly convex and $\tilde{\phi}$ is $(1 / \gamma)$-smooth. In addition, if $\phi$ is the vanilla hinge-loss, we have for every $a$ that

$$
\phi(a)-\gamma / 2 \leq \tilde{\phi}(a) \leq \phi(a)
$$

Max-of-hinge: The max-of-hinge loss function is a function from $\mathbb{R}^{k}$ to $\mathbb{R}$, which is defined as:

$$
\phi(a)=\max _{j}\left[c_{j}+a_{j}\right]_{+},
$$

for some $c \in \mathbb{R}^{k}$. This loss function is useful for multiclass prediction problems.

To calculate the conjugate of $\phi$, let

$$
S=\left\{\beta \in \mathbb{R}_{+}^{k}:\|\beta\|_{1} \leq 1\right\}
$$

and note that we can write $\phi$ as

$$
\phi(a)=\max _{\beta \in S} \sum_{j} \beta_{j}\left(c_{j}+a_{j}\right) .
$$

Hence, the conjugate of $\phi$ is

$$
\begin{aligned}
\phi^{*}(b) & =\max _{a}\left[a^{\top} b-\phi(a)\right]=\max _{a} \min _{\beta \in S}\left[a^{\top} b-\sum_{j} \beta_{j}\left(c_{j}+a_{j}\right)\right] \\
& =\min _{\beta \in S} \max _{a}\left[a^{\top} b-\sum_{j} \beta_{j}\left(c_{j}+a_{j}\right)\right]=\min _{\beta \in S}\left[-\sum_{j} \beta_{j} c_{j}+\sum_{j} \max _{a_{j}} a_{j}\left(b_{j}-\beta_{j}\right)\right] .
\end{aligned}
$$

Each inner maximization over $a_{j}$ would be $\infty$ unless $\beta_{j}=b_{j}$. Therefore,

$$
\phi^{*}(b)= \begin{cases}-c^{\top} b & \text { if } b \in S \\ \infty & \text { otherwise }\end{cases}
$$

Smooth max-of-hinge This loss obtained by smoothing the max-of-hinge loss using the technique described in Lemma2. This loss is parameterized by a scalar $\gamma>0$. We start by adding regularization to the conjugate of the max-of-hinge given in (11) and obtain

$$
\tilde{\phi}_{\gamma}^{*}(b)= \begin{cases}\frac{\gamma}{2}\|b\|^{2}-c^{\top} b & \text { if } b \in S \\ \infty & \text { otherwise }\end{cases}
$$


Taking the conjugate of the conjugate we obtain

$$
\begin{aligned}
\tilde{\phi}_{\gamma}(a) & =\max _{b} b^{\top} a-\tilde{\phi}_{\gamma}^{*}(b) \\
& =\max _{b \in S} b^{\top}(a+c)-\frac{\gamma}{2}\|b\|^{2} \\
& =\frac{\gamma}{2}\|(a+c) / \gamma\|^{2}-\frac{\gamma}{2} \min _{b \in S}\|b-(a+c) / \gamma\|^{2}
\end{aligned}
$$

While we do not have a closed form solution for the minimization problem over $b$ in the definition of $\tilde{\phi}_{\gamma}$ above, this is a problem of projecting onto the intersection of the $L_{1}$ ball and the positive orthant, and can be solved efficiently using the following procedure, adapted from [9].

\section{$\operatorname{Project}(\mu)$}

Goal: solve $\operatorname{argmin}_{b}\|b-\mu\|^{2}$ s.t. $b \in \mathbb{R}_{+}^{k},\|b\|_{1} \leq 1$

Let: $\forall i, \tilde{\mu}_{i}=\max \left\{0, \mu_{i}\right\}$

If: $\|\tilde{\mu}\|_{1} \leq 1$ stop and return $b=\tilde{\mu}$

Sort: let $i_{1}, \ldots, i_{k}$ be s.t. $\mu_{i_{1}} \geq \mu_{i_{2}} \geq \ldots \geq \mu_{i_{k}}$

Find: $j^{*}=\max \left\{j: j \tilde{\mu}_{i_{j}}+1-\sum_{r=1}^{j} \tilde{\mu}_{i_{r}}>0\right\}$

Define: $\theta=-1+\sum_{r=1}^{j^{*}} \tilde{\mu}_{i_{r}}$

Return: $b$ s.t. $\forall i, b_{i}=\max \left\{\mu_{i}-\theta / j^{*}, 0\right\}$

It also holds that $\nabla \tilde{\phi}_{\gamma}(a)=\operatorname{argmin}_{b \in S}\|b-(a+c) / \gamma\|^{2}$, and therefore the gradient can also be calculated using the above projection procedure.

Note that if $\phi$ being the max-of-hinge loss, then $\phi^{*}(b)+\gamma / 2 \geq \tilde{\phi}_{\gamma}^{*}(b) \geq \phi^{*}(b)$ and hence $\phi(a)-\gamma / 2 \leq$ $\tilde{\phi}_{\gamma}(a) \leq \phi(a)$.

Observe that all negative elements of $a+c$ does not contribute to $\tilde{\phi}_{\gamma}$. This immediately implies that if $\phi(a)=0$ then we also have $\tilde{\phi}_{\gamma}(a)=0$.

Soft-max-of-hinge loss function: Another approach to smooth the max-of-hinge loss function is by using soft-max instead of max. The resulting soft-max-of-hinge loss function is defined as

$$
\phi_{\gamma}(a)=\gamma \log \left(1+\sum_{i=1}^{k} e^{\left(c_{i}+a_{i}\right) / \gamma}\right)
$$

where $\gamma>0$ is a parameter. We have

$$
\max _{i}\left[c_{i}+a_{i}\right]_{+} \leq \phi_{\gamma}(a) \leq \max _{i}\left[c_{i}+a_{i}\right]_{+}+\gamma \log (k+1) .
$$

The $j$ 'th element of the gradient of $\phi$ is

$$
\nabla_{j} \phi_{\gamma}(a)=\frac{e^{\left(c_{j}+a_{j}\right) / \gamma}}{1+\sum_{i=1}^{k} e^{\left(c_{i}+a_{i}\right) / \gamma}} .
$$

By the definition of the conjugate we have $\phi_{\gamma}^{*}(b)=\max _{a} a^{\top} b-\phi_{\gamma}(a)$. The vector $a$ that maximizes the above must satisfy

$$
\forall j, \quad b_{j}=\frac{e^{\left(c_{j}+a_{j}\right) / \gamma}}{1+\sum_{i=1}^{k} e^{\left(c_{i}+a_{i}\right) / \gamma}} .
$$


This can be satisfied only if $b_{j} \geq 0$ for all $j$ and $\sum_{j} b_{j} \leq 1$. That is, $b \in S$. Denote $Z=\sum_{i=1}^{k} e^{\left(c_{i}+a_{i}\right) / \gamma}$ and note that

$$
(1+Z)\|b\|_{1}=Z \Rightarrow Z=\frac{\|b\|_{1}}{1-\|b\|_{1}} \Rightarrow 1+Z=\frac{1}{1-\|b\|_{1}} .
$$

It follows that

$$
a_{j}=\gamma\left(\log \left(b_{j}\right)+\log (1+Z)\right)-c_{j}=\gamma\left(\log \left(b_{j}\right)-\log \left(1-\|b\|_{1}\right)\right)-c_{j}
$$

which yields

$$
\begin{aligned}
\phi_{\gamma}^{*}(b) & =\sum_{j}\left(\gamma\left(\log \left(b_{j}\right)-\log \left(1-\|b\|_{1}\right)\right)-c_{j}\right) b_{j}+\gamma \log \left(1-\|b\|_{1}\right) \\
& =-c^{\top} b+\gamma\left(\left(1-\|b\|_{1}\right) \log \left(1-\|b\|_{1}\right)+\sum_{j} b_{j} \log \left(b_{j}\right)\right) .
\end{aligned}
$$

Finally, if $b \notin S$ then the gradient of $a^{\top} b-\phi_{\gamma}(a)$ does not vanish anywhere, which means that $\phi_{\gamma}^{*}(b)=\infty$. All in all, we obtain

$$
\phi_{\gamma}^{*}(b)= \begin{cases}-c^{\top} b+\gamma\left(\left(1-\|b\|_{1}\right) \log \left(1-\|b\|_{1}\right)+\sum_{j} b_{j} \log \left(b_{j}\right)\right) & \text { if } b \in S \\ \infty & \text { otherwise }\end{cases}
$$

Since the entropic function, $\sum_{j} b_{j} \log \left(b_{j}\right)$ is 1-strongly convex over $S$ with respect to the $L_{1}$ norm, we obtain that $\phi_{\gamma}^{*}$ is $\gamma$-strongly convex with respect to the $L_{1}$ norm, from which it follows that $\phi_{\gamma}$ is $(1 / \gamma)$-smooth with respect to the $L_{\infty}$ norm.

\subsection{Regularizers}

$L_{2}$ regularization: The simplest regularization is the squared $L_{2}$ regularization

$$
g(w)=\frac{1}{2}\|w\|_{2}^{2} .
$$

This is a 1-strongly convex regularization function whose conjugate is

$$
g^{*}(\theta)=\frac{1}{2}\|\theta\|_{2}^{2} .
$$

We also have

$$
\nabla g^{*}(\theta)=\theta .
$$

For our acceleration procedure, we also use the $L_{2}$ regularization plus a linear term, namely,

$$
g(w)=\frac{1}{2}\|w\|^{2}-w^{\top} z
$$

for some vector $z$. The conjugate of this function is

$$
g^{*}(\theta)=\max _{w}\left[w^{\top}(\theta+z)-\frac{1}{2}\|w\|^{2}\right]=\frac{1}{2}\|\theta+z\|^{2} .
$$

We also have

$$
\nabla g^{*}(\theta)=\theta+z
$$


$L_{1}$ regularization: Another popular regularization we consider is the $L_{1}$ regularization,

$$
f(w)=\sigma\|w\|_{1} .
$$

This is not a strongly convex regularizer and therefore we will add a slight $L_{2}$ regularization to it and define the $L_{1}-L_{2}$ regularization as

$$
g(w)=\frac{1}{2}\|w\|_{2}^{2}+\sigma^{\prime}\|w\|_{1},
$$

where $\sigma^{\prime}=\frac{\sigma}{\lambda}$ for some small $\lambda$. Note that

$$
\lambda g(w)=\frac{\lambda}{2}\|w\|_{2}^{2}+\sigma\|w\|_{1},
$$

so if $\lambda$ is small enough (as will be formalized later) we obtain that $\lambda g(w) \approx \sigma\|w\|_{1}$.

The conjugate of $g$ is

$$
g^{*}(v)=\max _{w}\left[w^{\top} v-\frac{1}{2}\|w\|_{2}^{2}-\sigma^{\prime}\|w\|_{1}\right] .
$$

The maximizer is also $\nabla g^{*}(v)$ and we now show how to calculate it. We have

$$
\begin{aligned}
\nabla g^{*}(v) & =\underset{w}{\operatorname{argmax}}\left[w^{\top} v-\frac{1}{2}\|w\|_{2}^{2}-\sigma^{\prime}\|w\|_{1}\right] \\
& =\underset{w}{\operatorname{argmin}}\left[\frac{1}{2}\|w-v\|_{2}^{2}+\sigma^{\prime}\|w\|_{1}\right]
\end{aligned}
$$

A sub-gradient of the objective of the optimization problem above is of the form $w-v+\sigma^{\prime} z=0$, where $z$ is a vector with $z_{i}=\operatorname{sign}\left(w_{i}\right)$, where if $w_{i}=0$ then $z_{i} \in[-1,1]$. Therefore, if $w$ is an optimal solution then for all $i$, either $w_{i}=0$ or $w_{i}=v_{i}-\sigma^{\prime} \operatorname{sign}\left(w_{i}\right)$. Furthermore, it is easy to verify that if $w$ is an optimal solution then for all $i$, if $w_{i} \neq 0$ then the sign of $w_{i}$ must be the sign of $v_{i}$. Therefore, whenever $w_{i} \neq 0$ we have that $w_{i}=v_{i}-\sigma^{\prime} \operatorname{sign}\left(v_{i}\right)$. It follows that in that case we must have $\left|v_{i}\right|>\sigma^{\prime}$. And, the other direction is also true, namely, if $\left|v_{i}\right|>\sigma^{\prime}$ then setting $w_{i}=v_{i}-\sigma^{\prime} \operatorname{sign}\left(v_{i}\right)$ leads to an objective value whose $i$ 'th component is

$$
\frac{1}{2}\left(\sigma^{\prime}\right)^{2}+\sigma^{\prime}\left(\left|v_{i}\right|-\sigma^{\prime}\right) \leq \frac{1}{2}\left|v_{i}\right|^{2},
$$

where the right-hand side is the $i$ 'th component of the objective value we will obtain by setting $w_{i}=0$. This leads to the conclusion that

$$
\nabla_{i} g^{*}(v)=\operatorname{sign}\left(v_{i}\right)\left[\left|v_{i}\right|-\sigma^{\prime}\right]_{+}= \begin{cases}v_{i}-\sigma^{\prime} \operatorname{sign}\left(v_{i}\right) & \text { if }\left|v_{i}\right|>\sigma^{\prime} \\ 0 & \text { o.w. }\end{cases}
$$

It follows that

$$
\begin{aligned}
g^{*}(v) & =\sum_{i} \operatorname{sign}\left(v_{i}\right)\left[\left|v_{i}\right|-\sigma^{\prime}\right]_{+} v_{i}-\frac{1}{2} \sum_{i}\left(\left[\left|v_{i}\right|-\sigma^{\prime}\right]_{+}\right)^{2}-\sigma^{\prime} \sum_{i}\left[\left|v_{i}\right|-\sigma^{\prime}\right]_{+} \\
& =\sum_{i}\left[\left|v_{i}\right|-\sigma^{\prime}\right]_{+}\left(\left|v_{i}\right|-\sigma^{\prime}-\frac{1}{2}\left[\left|v_{i}\right|-\sigma^{\prime}\right]_{+}\right) \\
& =\frac{1}{2} \sum_{i}\left(\left[\left|v_{i}\right|-\sigma^{\prime}\right]_{+}\right)^{2} .
\end{aligned}
$$


Another regularization function we'll use in the accelerated procedure is

$$
g(w)=\frac{1}{2}\|w\|_{2}^{2}+\sigma^{\prime}\|w\|_{1}-z^{\top} w .
$$

The conjugate function is

$$
g^{*}(v)=\frac{1}{2} \sum_{i}\left(\left[\left|v_{i}+z_{i}\right|-\sigma^{\prime}\right]_{+}\right)^{2}
$$

and its gradient is

$$
\nabla_{i} g^{*}(v)=\operatorname{sign}\left(v_{i}+z_{i}\right)\left[\left|v_{i}+z_{i}\right|-\sigma^{\prime}\right]_{+}
$$

\subsection{Ridge Regression}

In ridge regression, we minimize the squared loss with $L_{2}$ regularization. That is, $g(w)=\frac{1}{2}\|w\|^{2}$ and for every $i$ we have that $x_{i} \in \mathbb{R}^{d}$ and $\phi_{i}(a)=\frac{1}{2}\left(a-y_{i}\right)^{2}$ for some $y_{i} \in \mathbb{R}$. The primal problem is therefore

$$
P(w)=\frac{1}{2 n} \sum_{i=1}^{n}\left(x_{i}^{\top} w-y_{i}\right)^{2}+\frac{\lambda}{2}\|w\|^{2} .
$$

Below we specify Prox-SDCA for ridge regression. We use Option I since it is possible to derive a closed form solution to the maximization of the dual with respect to $\Delta \alpha_{i}$. Indeed, since $-\phi_{i}^{*}(-b)=-\frac{1}{2} b^{2}+y_{i} b$ we have that the maximization problem is

$$
\begin{aligned}
\Delta \alpha_{i} & =\underset{b}{\operatorname{argmax}}-\frac{1}{2}\left(\alpha_{i}^{(t+1)}+b\right)^{2}+y_{i}\left(\alpha_{i}^{(t+1)}+b\right)-w^{(t-1) \top} x_{i} b-\frac{b^{2}\left\|x_{i}\right\|^{2}}{2 \lambda n} \\
& =\underset{b}{\operatorname{argmax}}-\frac{1}{a}\left(1+\frac{\left\|x_{i}\right\|^{2}}{2 \lambda n}\right) b^{2}-\left(\alpha_{i}^{(t+1)}+w^{(t-1) \top} x_{i}-y_{i}\right) b \\
& =-\frac{\alpha_{i}^{(t+1)}+w^{(t-1) \top} x_{i}-y_{i}}{1+\frac{\left\|x_{i}\right\|^{2}}{2 \lambda n}} .
\end{aligned}
$$

Applying the above update and using some additional tricks to improve the running time we obtain the following procedure.

Prox-SDCA $\left(\left(x_{i}, y_{i}\right)_{i=1}^{n}, \epsilon, \alpha^{(0)}, z\right)$ for solving ridge regression

Goal: Minimize $P(w)=\frac{1}{2 n} \sum_{i=1}^{n}\left(x_{i}^{\top} w-y_{i}\right)^{2}+\lambda\left(\frac{1}{2}\|w\|^{2}-w^{\top} z\right)$

Initialize $v^{(0)}=\frac{1}{\lambda n} \sum_{i=1}^{n} \alpha_{i}^{(0)} x_{i}, \forall i, \tilde{y}_{i}=y_{i}-x_{i}^{\top} z$

Iterate: for $t=1,2, \ldots$

Randomly pick $i$

$$
\begin{aligned}
& \Delta \alpha_{i}=-\frac{\alpha_{i}^{(t-1)}+v^{(t-1) \top} x_{i}-\tilde{y}_{i}}{1+\frac{\left\|x_{i}\right\|^{2}}{2 \lambda n}} \\
& \alpha_{i}^{(t)} \leftarrow \alpha_{i}^{(t-1)}+\Delta \alpha_{i} \text { and for } j \neq i, \alpha_{j}^{(t)} \leftarrow \alpha_{j}^{(t-1)} \\
& v^{(t)} \leftarrow v^{(t-1)}+\frac{\Delta \alpha_{i}}{\lambda n} x_{i}
\end{aligned}
$$

\section{Stopping condition:}

Let $w^{(t)}=v^{(t)}+z$

Stop if $\frac{1}{2 n} \sum_{i=1}^{n}\left(\left(x_{i}^{\top} w^{(t)}-y_{i}\right)^{2}+\left(\alpha_{i}^{(t)}+y_{i}\right)^{2}-y_{i}^{2}\right)+\lambda w^{(t)^{\top}} v^{(t)} \leq \epsilon$ 
The runtime of Prox-SDCA for ridge regression becomes

$$
\tilde{O}\left(d\left(n+\frac{R^{2}}{\lambda}\right)\right)
$$

where $R=\max _{i}\left\|x_{i}\right\|$. This matches the recent results of [15, 25]. If $R^{2} / \lambda \gg n$ we can apply the accelerated procedure and obtain the improved runtime

$$
\tilde{O}\left(d \sqrt{\frac{n R^{2}}{\lambda}}\right) .
$$

\subsection{Logistic Regression}

In logistic regression, we minimize the logistic loss with $L_{2}$ regularization. That is, $g(w)=\frac{1}{2}\|w\|^{2}$ and for every $i$ we have that $x_{i} \in \mathbb{R}^{d}$ and $\phi_{i}(a)=\log \left(1+e^{a}\right)$. The primal problem is therefore ${ }^{3}$

$$
P(w)=\frac{1}{n} \sum_{i=1}^{n} \log \left(1+e^{x_{i}^{\top} w}\right)+\frac{\lambda}{2}\|w\|^{2} .
$$

The dual problem is

$$
D(\alpha)=\frac{1}{n} \sum_{i=1}^{n}\left(\alpha_{i} \log \left(-\alpha_{i}\right)-\left(1+\alpha_{i}\right) \log \left(1+\alpha_{i}\right)\right)-\frac{\lambda}{2}\|v(\alpha)\|^{2},
$$

and the dual constraints are $\alpha \in[-1,0]^{n}$.

Below we specify Prox-SDCA for logistic regression using Option III.

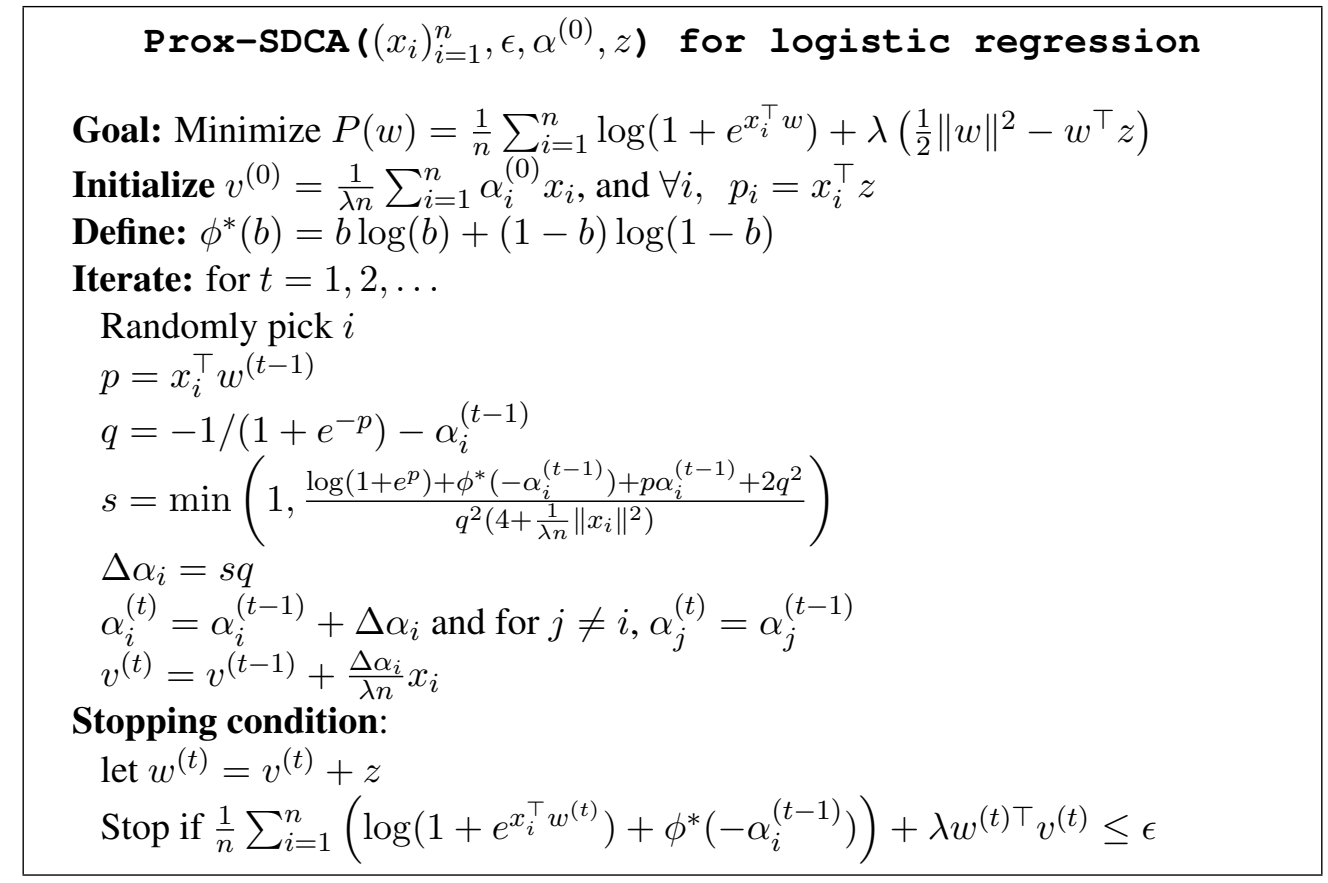

The runtime analysis is similar to the analysis for ridge regression.

\footnotetext{
${ }^{3}$ Usually, the training data comes with labels, $y_{i} \in\{ \pm 1\}$, and the loss function becomes $\log \left(1+e^{-y_{i} x_{i}^{\top} w}\right)$. However, we can easily get rid of the labels by re-defining $x_{i} \leftarrow-y_{i} x_{i}$.
} 


\subsection{Lasso}

In the Lasso problem, the loss function is the squared loss but the regularization function is $L_{1}$. That is, we need to solve the problem:

$$
\min _{w}\left[\frac{1}{2 n} \sum_{i=1}^{n}\left(x_{i}^{\top} w-y_{i}\right)^{2}+\sigma\|w\|_{1}\right],
$$

with a positive regularization parameter $\sigma \in \mathbb{R}_{+}$.

Let $\bar{y}=\frac{1}{2 n} \sum_{i=1}^{n} y_{i}^{2}$, and let $\bar{w}$ be an optimal solution of (18). Then, the objective at $\bar{w}$ is at most the objective at $w=0$, which yields

$$
\sigma\|\bar{w}\|_{1} \leq \bar{y} \Rightarrow\|\bar{w}\|_{2} \leq\|\bar{w}\|_{1} \leq \frac{\bar{y}}{\sigma}
$$

Consider the optimization problem

$$
\min _{w} P(w) \text { where } P(w)=\frac{1}{2 n} \sum_{i=1}^{n}\left(x_{i}^{\top} w-y_{i}\right)^{2}+\lambda\left(\frac{1}{2}\|w\|_{2}^{2}+\frac{\sigma}{\lambda}\|w\|_{1}\right),
$$

for some $\lambda>0$. This problem fits into our framework, since now the regularizer is strongly convex. Furthermore, if $w^{*}$ is an $(\epsilon / 2)$-accurate solution to the problem in $(19)$, then $P\left(w^{*}\right) \leq P(\bar{w})+\epsilon / 2$ which yields

$$
\left[\frac{1}{2 n} \sum_{i=1}^{n}\left(x_{i}^{\top} w^{*}-y_{i}\right)^{2}+\sigma\left\|w^{*}\right\|_{1}\right] \leq\left[\frac{1}{2 n} \sum_{i=1}^{n}\left(x_{i}^{\top} \bar{w}-y_{i}\right)^{2}+\sigma\|\bar{w}\|_{1}\right]+\frac{\lambda}{2}\|\bar{w}\|_{2}^{2}+\epsilon / 2 .
$$

Since $\|\bar{w}\|_{2}^{2} \leq(\bar{y} / \sigma)^{2}$, we obtain that setting $\lambda=\epsilon(\sigma / \bar{y})^{2}$ guarantees that $w^{*}$ is an $\epsilon$ accurate solution to the original problem given in 18 .

In light of the above, from now on we focus on the problem given in (19). As in the case of ridge regression, we can apply Prox-SDCA with Option I. The resulting pseudo-code is given below. Applying the above update and using some additional tricks to improve the running time we obtain the following procedure.

Prox-SDCA $\left(\left(x_{i}, y_{i}\right)_{i=1}^{n}, \epsilon, \alpha^{(0)}, z\right)$ for solving $L_{1}-L_{2}$ regression

Goal: Minimize $P(w)=\frac{1}{2 n} \sum_{i=1}^{n}\left(x_{i}^{\top} w-y_{i}\right)^{2}+\lambda\left(\frac{1}{2}\|w\|^{2}+\sigma^{\prime}\|w\|_{1}-w^{\top} z\right)$

Initialize $v^{(0)}=\frac{1}{\lambda n} \sum_{i=1}^{n} \alpha_{i}^{(0)} x_{i}$, and $\forall j, w_{j}^{(0)}=\operatorname{sign}\left(v_{j}^{(0)}+z_{j}\right)\left[\left|v_{j}^{(0)}+z_{j}\right|-\sigma^{\prime}\right]_{+}$ Iterate: for $t=1,2, \ldots$

Randomly pick $i$

$$
\begin{aligned}
& \Delta \alpha_{i}=-\frac{\alpha_{i}^{(t-1)}+w^{(t-1) \top} x_{i}-y_{i}}{1+\frac{\left\|x_{i}\right\|^{2}}{2 \lambda n}} \\
& \alpha_{i}^{(t)}=\alpha_{i}^{(t-1)}+\Delta \alpha_{i} \text { and for } j \neq i, \alpha_{j}^{(t)}=\alpha_{j}^{(t-1)} \\
& v^{(t)}=v^{(t-1)}+\frac{\Delta \alpha_{i}}{\lambda n} x_{i} \\
& \forall j, w_{j}^{(t)}=\operatorname{sign}\left(v_{j}^{(t)}+z_{j}\right)\left[\left|v_{j}^{(t)}+z_{j}\right|-\sigma^{\prime}\right]_{+}
\end{aligned}
$$

\section{Stopping condition:}

$$
\text { Stop if } \frac{1}{2 n} \sum_{i=1}^{n}\left(\left(x_{i}^{\top} w^{(t)}-y_{i}\right)^{2}-2 y_{i} \alpha_{i}^{(t)}+\left(\alpha_{i}^{(t)}\right)^{2}\right)+\lambda w^{(t) \top} v^{(t)} \leq \epsilon
$$


Let us now discuss the runtime of the resulting method. Denote $R=\max _{i}\left\|x_{i}\right\|$ and for simplicity, assume that $\bar{y}=O(1)$. Choosing $\lambda=\epsilon(\sigma / \bar{y})^{2}$, the runtime of our method becomes

$$
\tilde{O}\left(d\left(n+\min \left\{\frac{R^{2}}{\epsilon \sigma^{2}}, \sqrt{\frac{n R^{2}}{\epsilon \sigma^{2}}}\right\}\right)\right) .
$$

It is also convenient to write the bound in terms of $B=\|\bar{w}\|_{2}$, where, as before, $\bar{w}$ is the optimal solution of the $L_{1}$ regularized problem. With this parameterization, we can set $\lambda=\epsilon / B^{2}$ and the runtime becomes

$$
\tilde{O}\left(d\left(n+\min \left\{\frac{R^{2} B^{2}}{\epsilon}, \sqrt{\frac{n R^{2} B^{2}}{\epsilon}}\right\}\right)\right) .
$$

The runtime of standard SGD is $O\left(d R^{2} B^{2} / \epsilon^{2}\right)$ even in the case of smooth loss functions such as the squared loss. Several variants of SGD, that leads to sparser intermediate solutions, have been proposed (e.g. [14, 21, 27, 8, 10] $)$. However, all of these variants share the runtime of $O\left(d R^{2} B^{2} / \epsilon^{2}\right)$, which is much slower than our runtime when $\epsilon$ is small.

Another relevant approach is the FISTA algorithm of [2]. The shrinkage operator of FISTA is the same as the gradient of $g^{*}$ used in our approach. It is a batch algorithm using Nesterov's accelerated gradient technique. For the squared loss function, the runtime of FISTA is

$$
O\left(d n \sqrt{\frac{R^{2} B^{2}}{\epsilon}}\right) .
$$

This bound is worst than our bound by a factor of at least $\sqrt{n}$.

Another approach to solving [18] is stochastic coordinate descent over the primal problem. [21] showed that the runtime of this approach is

$$
O\left(\frac{d n B^{2}}{\epsilon}\right)
$$

under the assumption that $\left\|x_{i}\right\|_{\infty} \leq 1$ for all $i$. Similar results can also be found in [16].

For our method, the runtime depends on $R^{2}=\max _{i}\left\|x_{i}\right\|_{2}^{2}$. If $R^{2}=O(1)$ then the runtime of our method is much better than that of [21]. In the general case, if $\max _{i}\left\|x_{i}\right\|_{\infty} \leq 1$ then $R^{2} \leq d$, which yields the runtime of

$$
\tilde{O}\left(d\left(n+\min \left\{\frac{d B^{2}}{\epsilon}, \sqrt{\frac{n d B^{2}}{\epsilon}}\right\}\right)\right) .
$$

This is the same or better than [21] whenever $d=O(n)$.

\subsection{Linear SVM}

Support Vector Machines (SVM) is an algorithm for learning a linear classifier. Linear SVM (i.e., SVM with linear kernels) amounts to minimizing the objective

$$
P(w)=\frac{1}{n} \sum_{i=1}^{n}\left[1-x_{i}^{\top} w\right]_{+}+\frac{\lambda}{2}\|w\|^{2},
$$

where $[a]_{+}=\max \{0, a\}$, and for every $i, x_{i} \in \mathbb{R}^{d}$. This can be cast as the objective given in (1) by letting the regularization be $g(w)=\frac{1}{2}\|w\|_{2}^{2}$, and for every $i, \phi_{i}(a)=[1-a]_{+}$, is the hinge-loss. 
Let $R=\max _{i}\left\|x_{i}\right\|_{2}$. SGD enjoys the rate of $O\left(\frac{1}{\lambda \epsilon}\right)$. Many software packages apply SDCA and obtain the rate $\tilde{O}\left(n+\frac{1}{\lambda \epsilon}\right)$. We now show how our accelerated proximal SDCA enjoys the rate $\tilde{O}\left(n+\sqrt{\frac{n}{\lambda \epsilon}}\right)$. This is significantly better than the rate of SGD when $\lambda \epsilon<1 / n$. We note that a default setting for $\lambda$, which often works well in practice, is $\lambda=1 / n$. In this case, $\lambda \epsilon=\epsilon / n \ll 1 / n$.

Our first step is to smooth the hinge-loss. Let $\gamma=\epsilon$ and consider the smooth hinge-loss as defined in 9). Recall that the smooth hinge-loss satisfies

$$
\forall a, \quad \phi(a)-\gamma / 2 \leq \tilde{\phi}(a) \leq \phi(a) .
$$

Let $\tilde{P}$ be the SVM objective while replacing the hinge-loss with the smooth hinge-loss. Therefore, for every $w^{\prime}$ and $w$,

$$
P\left(w^{\prime}\right)-P(w) \leq \tilde{P}\left(w^{\prime}\right)-\tilde{P}(w)+\gamma / 2 .
$$

It follows that if $w^{\prime}$ is an $(\epsilon / 2)$-optimal solution for $\tilde{P}$, then it is $\epsilon$-optimal solution for $P$.

For the smoothed hinge loss, the optimization problem given in Option I of Prox-SDCA has a closed form solution and we obtain the following procedure:

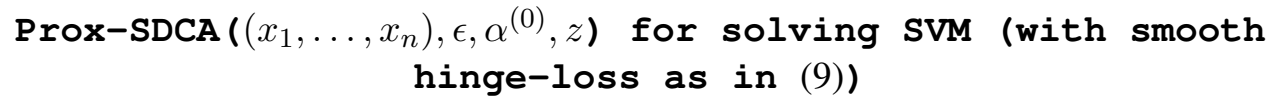

Define: $\tilde{\phi}_{\gamma}$ as in 9 )

Goal: Minimize $P(w)=\frac{1}{n} \sum_{i=1}^{n} \tilde{\phi}_{\gamma}\left(x_{i}^{\top} w\right)+\lambda\left(\frac{1}{2}\|w\|^{2}-w^{\top} z\right)$

Initialize $w^{(0)}=z+\frac{1}{\lambda n} \sum_{i=1}^{n} \alpha_{i}^{(0)} x_{i}$

Iterate: for $t=1,2, \ldots$

Randomly pick $i$

$\Delta \alpha_{i}=\max \left(-\alpha_{i}^{(t-1)}, \min \left(1-\alpha_{i}^{(t-1)}, \frac{1-x_{i}^{\top} w^{(t-1)}-\gamma \alpha_{i}^{(t-1)}}{\left\|x_{i}\right\|^{2} /(\lambda n)+\gamma}\right)\right)$

$\alpha_{i}^{(t)} \leftarrow \alpha_{i}^{(t-1)}+\Delta \alpha_{i}$ and for $j \neq i, \alpha_{j}^{(t)} \leftarrow \alpha_{j}^{(t-1)}$

$w^{(t)} \leftarrow w^{(t-1)}+\frac{\Delta \alpha_{i}}{\lambda n} x_{i}$

\section{Stopping condition:}

Stop if $\frac{1}{n} \sum_{i=1}^{n}\left(\tilde{\phi}_{\gamma}\left(x_{i}^{\top} w^{(t)}\right)-\alpha_{i}^{(t)}+\frac{\gamma}{2}\left(\alpha_{i}^{(t)}\right)^{2}\right)+\lambda w^{(t)^{\top}}\left(w^{(t)}-z\right) \leq \epsilon$

Denote $R=\max _{i}\left\|x_{i}\right\|$. Then, the runtime of the resulting method is

$$
\tilde{O}\left(d\left(n+\min \left\{\frac{R^{2}}{\gamma \lambda}, \sqrt{\frac{n R^{2}}{\gamma \lambda}}\right\}\right)\right) .
$$

In particular, choosing $\gamma=\epsilon$ we obtain a solution to the original SVM problem in runtime of

$$
\tilde{O}\left(d\left(n+\min \left\{\frac{R^{2}}{\epsilon \lambda}, \sqrt{\frac{n R^{2}}{\epsilon \lambda}}\right\}\right)\right) .
$$

As mentioned before, this is better than SGD when $\frac{1}{\lambda \epsilon} \gg n$. 


\subsection{Multiclass SVM}

Next we consider Multiclass SVM using the construction described in Crammer and Singer [5]. Each example consists of an instance vector $x_{i} \in \mathbb{R}^{d}$ and a label $y_{i} \in\{1, \ldots, k\}$. The goal is to learn a matrix $W \in \mathbb{R}^{d, k}$ such that $W^{\top} x_{i}$ is a $k$ 'th dimensional vector of scores for the different classes. The prediction is the coordinate of $W^{\top} x_{i}$ of maximal value. The loss function is

$$
\max _{j \neq y_{i}}\left(1+\left(W^{\top} x_{i}\right)_{j}-\left(W^{\top} x_{i}\right)_{y_{i}}\right) .
$$

This can be written as $\phi\left(\left(W^{\top} x_{i}\right)-\left(W^{\top} x_{i}\right)_{y_{i}}\right)$ where

$$
\phi_{i}(a)=\max _{j}\left[c_{i, j}+a_{j}\right]_{+},
$$

with $c_{i}$ being the all ones vector except 0 in the $y_{i}$ coordinate.

We can model this in our framework as follows. Given a matrix $M$ let $\operatorname{vec}(M)$ be the column vector obtained by concatenating the columns of $M$. Let $e_{j}$ be the all zeros vector except 1 in the $j$ 'th coordinate. For every $i$, let $c_{i}=\mathbf{1}-e_{y_{i}}$ and let $X_{i} \in \mathbb{R}^{d k, k}$ be the matrix whose $j$ 'th column is $\operatorname{vec}\left(x_{i}\left(e_{j}-e_{y_{i}}\right)^{\top}\right)$. Then,

$$
X_{i}^{\top} \operatorname{vec}(W)=W^{\top} x_{i}-\left(W^{\top} x_{i}\right)_{y_{i}} .
$$

Therefore, the optimization problem of multiclass SVM becomes:

$$
\min _{w \in \mathbb{R}^{d k}} P(w) \text { where } P(w)=\frac{1}{n} \sum_{i=1}^{n} \phi_{i}\left(X_{i}^{\top} w\right)+\frac{\lambda}{2}\|w\|^{2} .
$$

As in the case of SVM, we will use the smooth version of the max-of-hinge loss function as described in (13). If we set the smoothness parameter $\gamma$ to be $\epsilon$ then an $(\epsilon / 2)$-accurate solution to the problem with the smooth loss is also an $\epsilon$-accurate solution to the original problem with the non-smooth loss. Therefore, from now on we focus on the problem with the smooth max-of-hinge loss.

We specify Prox-SDCA for multiclass SVM using Option I. We will show that the optimization problem in Option I can be calculated efficiently by sorting a $k$ dimensional vector. Such ideas were explored in [5] for the non-smooth max-of-hinge loss.

Let $\hat{w}=w-\frac{1}{\lambda n} X_{i} \alpha_{i}^{(t-1)}$. Then, the optimization problem over $\alpha_{i}$ can be written as

$$
\underset{\alpha_{i}:-\alpha_{i} \in S}{\operatorname{argmax}}\left(-c_{i}^{\top}-\hat{w}^{\top} X_{i}\right) \alpha_{i}-\frac{\gamma}{2}\left\|\alpha_{i}\right\|^{2}-\frac{1}{2 \lambda n}\left\|X_{i} \alpha_{i}\right\|^{2} .
$$

As shown before, if we organize $\hat{w}$ as a $d \times k$ matrix, denoted $\hat{W}$, we have that $X_{i}^{\top} \hat{w}=\hat{W}^{\top} x_{i}-\left(\hat{W}^{\top} x_{i}\right)_{y_{i}}$. We also have that

$$
X_{i} \alpha_{i}=\sum_{j} \operatorname{vec}\left(x_{i}\left(e_{j}-e_{y_{i}}\right)^{\top}\right) \alpha_{i, j}=\operatorname{vec}\left(x_{i} \sum_{j} \alpha_{i, j}\left(e_{j}-e_{y_{i}}\right)^{\top}\right)=\operatorname{vec}\left(x_{i}\left(\alpha_{i}-\left\|\alpha_{i}\right\|_{1} e_{y_{i}}\right)^{\top}\right) .
$$

It follows that an optimal solution to (20) must set $\alpha_{i, y_{i}}=0$ and we only need to optimize over the rest of the dual variables. This also yields,

$$
\left\|X_{i} \alpha_{i}\right\|^{2}=\left\|x_{i}\right\|^{2}\left\|\alpha_{i}\right\|_{2}^{2}+\left\|x_{i}\right\|^{2}\left\|\alpha_{i}\right\|_{1}^{2} .
$$


So, 20, becomes:

$$
\underset{\alpha_{i}:-\alpha_{i} \in S, \alpha_{i, y_{i}}=0}{\operatorname{argmax}}\left(-c_{i}^{\top}-\hat{w}^{\top} X_{i}\right) \alpha_{i}-\frac{\gamma}{2}\left\|\alpha_{i}\right\|_{2}^{2}-\frac{\left\|x_{i}\right\|^{2}}{2 \lambda n}\left\|\alpha_{i}\right\|_{2}^{2}-\frac{\left\|x_{i}\right\|^{2}}{2 \lambda n}\left\|\alpha_{i}\right\|_{1}^{2} .
$$

This is equivalent to a problem of the form:

$$
\underset{a \in \mathbb{R}_{+}^{k-1}, \beta}{\operatorname{argmin}}\|a-\mu\|_{2}^{2}+C \beta^{2} \text { s.t. }\|a\|_{1}=\beta \leq 1,
$$

where

$$
\mu=\frac{c_{i}^{\top}+\hat{w}^{\top} X_{i}}{\gamma+\frac{\left\|x_{i}\right\|^{2}}{\lambda n}} \text { and } C=\frac{\frac{\left\|x_{i}\right\|^{2}}{\lambda n}}{\gamma+\frac{\left\|x_{i}\right\|^{2}}{\lambda n}}=\frac{1}{\frac{\gamma \lambda n}{\left\|x_{i}\right\|^{2}}+1} .
$$

The equivalence is in the sense that if $(a, \beta)$ is a solution of (22) then we can set $\alpha_{i}=-a$.

Assume for simplicity that $\mu$ is sorted in a non-increasing order and that all of its elements are nonnegative (otherwise, it is easy to verify that we can zero the negative elements of $\mu$ and sort the non-negative, without affecting the solution). Let $\bar{\mu}$ be the cumulative sum of $\mu$, that is, for every $j$, let $\bar{\mu}_{j}=\sum_{r=1}^{j} \mu_{r}$. For every $j$, let $z_{j}=\bar{\mu}_{j}-j \mu_{j}$. Since $\mu$ is sorted we have that

$$
z_{j+1}=\sum_{r=1}^{j+1} \mu_{r}-(j+1) \mu_{j+1}=\sum_{r=1}^{j} \mu_{r}-j \mu_{j+1} \geq \sum_{r=1}^{j} \mu_{r}-j \mu_{j}=z_{j} .
$$

Note also that $z_{1}=0$ and that $z_{k}=\bar{\mu}_{k}=\|\mu\|_{1}$ (since the coordinate of $\mu$ that corresponds to $y_{i}$ is zero). By the properties of projection onto the simplex (see [9]), for every $z \in\left(z_{j}, z_{j+1}\right)$ we have that the projection of $\mu$ onto the set $\left\{b \in \mathbb{R}_{+}^{k}:\|b\|_{1}=z\right\}$ is of the form $a_{r}=\max \left\{0, \mu_{r}-\theta / j\right\}$ where $\theta=\left(-z+\bar{\mu}_{j}\right) / j$. Therefore, the objective becomes (ignoring constants that do not depend on $z$ ),

$$
j \theta^{2}+C z^{2}=\left(-z+\bar{\mu}_{j}\right)^{2} / j+C z^{2} .
$$

The first order condition for minimality w.r.t. $z$ is

$$
-\left(-z+\bar{\mu}_{j}\right) / j+C z=0 \Rightarrow z=\frac{\bar{\mu}_{j}}{1+j C} .
$$

If this value of $z$ is in $\left(z_{j}, z_{j+1}\right)$, then it is the optimal $z$ and we're done. Otherwise, the optimum should be either $z=0$ (which yields $\alpha=0$ as well) or $z=1$. 


$$
a=\text { OptimizeDual }(\mu, C)
$$

Solve the optimization problem given in 22)

Initialize: $\forall i, \hat{\mu}_{i}=\max \left\{0, \mu_{i}\right\}$, and sort $\hat{\mu}$ s.t. $\hat{\mu}_{1} \geq \hat{\mu}_{2} \geq \ldots \geq \hat{\mu}_{k}$

Let: $\bar{\mu}$ be s.t. $\bar{\mu}_{j}=\sum_{i=1}^{j} \hat{\mu}_{i}$

Let: $z$ be s.t. $z_{j}=\min \left\{\bar{\mu}_{j}-j \hat{\mu}_{j}, 1\right\}$ and $z_{k+1}=1$

If: $\exists j$ s.t. $\frac{\bar{\mu}_{j}}{1+j C} \in\left[z_{j}, z_{j+1}\right]$ return $a$ s.t. $\forall i, a_{i}=\max \left\{0, \mu_{i}-\left(-\frac{\bar{\mu}_{j}}{1+j C}+\bar{\mu}_{j}\right) / j\right\}$

\section{Else:}

Let $j$ be the minimal index s.t. $z_{j}=1$

set $a$ s.t. $\forall i, a_{i}=\max \left\{0, \mu_{i}-\left(-z_{j}+\bar{\mu}_{j}\right) / j\right\}$

If: $\|a-\mu\|^{2}+C \leq\|\mu\|^{2}$ return $a$

Else:

return $(0, \ldots, 0)$

The resulting pseudo-codes for Prox-SDCA is given below. We specify the procedure while referring to $W$ as a matrix, because it is the more natural representation. For convenience of the code, we also maintain in $\alpha_{i, y_{i}}$ the value of $-\sum_{j \neq y_{i}} \alpha_{i, j}$ (instead of the optimal value of 0 ).

\section{Prox-SDCA $\left(\left(x_{1}, y_{1}\right)_{i=1}^{n}, \epsilon, \alpha, Z\right)$ for solving Multiclass SVM \\ (with smooth hinge-loss as in (13))}

Define: $\tilde{\phi}_{\gamma}$ as in (13)

Goal: Minimize

$P(W)=\frac{1}{n} \sum_{i=1}^{n} \tilde{\phi}_{\gamma}\left(\left(W^{\top} x_{i}\right)-\left(W^{\top} x_{i}\right)_{y_{i}}\right)+\lambda\left(\frac{1}{2} \operatorname{vec}(W)^{\top} \operatorname{vec}(W)-\operatorname{vec}(W)^{\top} \operatorname{vec}(Z)\right)$

Initialize $W=Z+\frac{1}{\lambda n} \sum_{i=1}^{n} x_{i} \alpha_{i}^{\top}$

Iterate: for $t=1,2, \ldots$

Randomly pick $i$

$\hat{W}=W-\frac{1}{\lambda n} x_{i} \alpha_{i}^{\top}$

$p=x_{i}^{\top} \hat{W}, \stackrel{p}{p}=p-p_{y_{i}}, c=\mathbf{1}-e_{y_{i}}, \quad \mu=\frac{c+p}{\gamma+\left\|x_{i}\right\|^{2} /(\lambda n)}, C=\frac{1}{1+\gamma \lambda n /\left\|x_{i}\right\|^{2}}$

$a=\operatorname{OptimizeDual}(\mu, C)$

$\alpha_{i}=-a, \alpha_{y_{i}}=\|a\|_{1}$

$W=\hat{W}+\frac{1}{\lambda n} x_{i} \alpha_{i}^{\top}$

\section{Stopping condition:}

let $G=0$

for $i=1, \ldots, n$

$a=W^{\top} x_{i}, a=a-a_{y_{i}}, c=\mathbf{1}-e_{y_{i}}, b=\operatorname{Project}((a+c) / \gamma)$

$G=G+\frac{\gamma}{2}\left(\|(a+c) / \gamma\|^{2}-\|b-(a+c) / \gamma\|^{2}\right)+c^{\top} \alpha_{i}^{(t)}+\frac{\gamma}{2}\left(\left\|\alpha_{i}^{(t)}\right\|^{2}-\left(\alpha_{i, y_{i}}^{(t)}\right)^{2}\right)$

Stop if $G / n+\lambda \operatorname{vec}(W)^{\top} \operatorname{vec}(W-Z) \leq \epsilon$ 


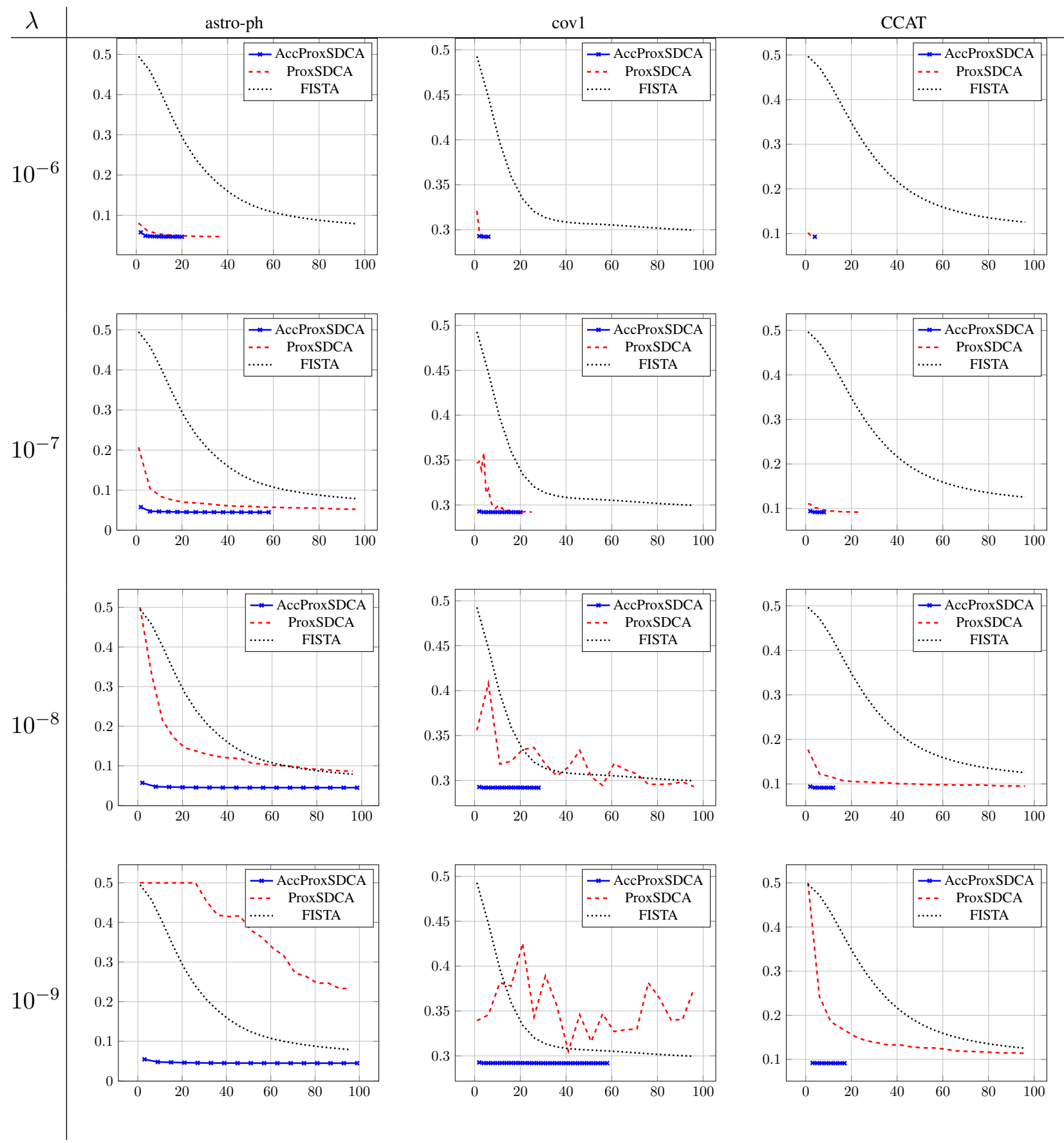

Figure 3: Comparing Accelerated-Prox-SDCA, Prox-SDCA, and FISTA for minimizing the smoothed hinge-loss $(\gamma=1)$ with $L_{1}-L_{2}$ regularization $\left(\sigma=10^{-5}\right.$ and $\lambda$ varies in $\left.\left\{10^{-6}, \ldots, 10^{-9}\right\}\right)$. In each of these plots, the $\mathrm{y}$-axis is the primal objective and the $\mathrm{x}$-axis is the number of passes through the entire training set. The three columns corresponds to the three data sets. The methods are terminated either if stopping condition is met (with $\epsilon=10^{-3}$ ) or after 100 passes over the data. 


\section{Experiments}

In this section we compare Prox-SDCA, its accelerated version Accelerated-Prox-SDCA, and the FISTA algorithm of [2], on $L_{1}-L_{2}$ regularized loss minimization problems.

The experiments were performed on three large datasets with very different feature counts and sparsity, which were kindly provided by Thorsten Joachims (the datasets were also used in [24]). The astro-ph dataset classifies abstracts of papers from the physics ArXiv according to whether they belong in the astrophysics section; CCAT is a classification task taken from the Reuters RCV1 collection; and cov1 is class 1 of the covertype dataset of Blackard, Jock \& Dean. The following table provides details of the dataset characteristics.

\begin{tabular}{|r|c|c|c|c|}
\hline Dataset & Training Size & Testing Size & Features & Sparsity \\
\hline astro-ph & 29882 & 32487 & 99757 & $0.08 \%$ \\
CCAT & 781265 & 23149 & 47236 & $0.16 \%$ \\
cov1 & 522911 & 58101 & 54 & $22.22 \%$ \\
\hline
\end{tabular}

These are binary classification problems, with each $x_{i}$ being a vector which has been normalized to be $\left\|x_{i}\right\|_{2}=1$, and $y_{i}$ being a binary class label of \pm 1 . We multiplied each $x_{i}$ by $y_{i}$ and following [24], we employed the smooth hinge loss, $\tilde{\phi}_{\gamma}$, as in (9), with $\gamma=1$. The optimization problem we need to solve is therefore

$$
\min _{w} P(w) \text { where } P(w)=\frac{1}{n} \sum_{i=1}^{n} \tilde{\phi}_{\gamma}\left(x_{i}^{\top} w\right)+\frac{\lambda}{2}\|w\|_{2}^{2}+\sigma\|w\|_{1} .
$$

In the experiments, we set $\sigma=10^{-5}$ and vary $\lambda$ in the range $\left\{10^{-6}, 10^{-7}, 10^{-8}, 10^{-9}\right\}$.

The convergence behaviors are plotted in Figure 3. In all the plots we depict the primal objective as a function of the number of passes over the data (often referred to as "epochs"). For FISTA, each iteration involves a single pass over the data. For Prox-SDCA, each $n$ iterations are equivalent to a single pass over the data. And, for Accelerated-Prox-SDCA, each $n$ inner iterations are equivalent to a single pass over the data. For Prox-SDCA and Accelerated-Prox-SDCA we implemented their corresponding stopping conditions and terminate the methods once an accuracy of $10^{-3}$ was guaranteed.

It is clear from the graphs that Accelerated-Prox-SDCA yields the best results, and often significantly outperform the other methods. Prox-SDCA behaves similarly when $\lambda$ is relatively large, but it converges much slower when $\lambda$ is small. This is consistent with our theory. Finally, the relative performance of FISTA and Prox-SDCA depends on the ratio between $\lambda$ and $n$, but in all cases, Accelerated-Prox-SDCA is much faster than FISTA. This is again consistent with our theory.

\section{Discussion and Open Problems}

We have described and analyzed a proximal stochastic dual coordinate ascent method and have shown how to accelerate the procedure. The overall runtime of the resulting method improves state-of-the-art results in many cases of interest.

There are two main open problems that we leave to future research.

Open Problem 1. When $\frac{1}{\lambda \gamma}$ is larger than $n$, the runtime of our procedure becomes $\tilde{O}\left(d \sqrt{\frac{n}{\lambda \gamma}}\right)$. Is it possible to derive a method whose runtime is $\tilde{O}\left(d\left(n+\sqrt{\frac{1}{\lambda \gamma}}\right)\right)$ ? 
Open Problem 2. Our Prox-SDCA procedure and its analysis works for regularizers which are strongly convex with respect to an arbitrary norm. However, our acceleration procedure is designed for regularizers which are strongly convex with respect to the Euclidean norm. Is is possible to extend the acceleration procedure to more general regularizers?

\section{Acknowledgements}

The authors would like to thank Fen Xia for careful proof-reading of the paper which helped us to correct numerous typos. Shai Shalev-Shwartz is supported by the following grants: Intel Collaborative Research Institute for Computational Intelligence (ICRI-CI) and ISF 598-10. Tong Zhang is supported by the following grants: NSF IIS-1016061, NSF DMS-1007527, and NSF IIS-1250985.

\section{A Proofs of Iteration Bounds for Prox-SDCA}

The proof technique follows that of Shalev-Shwartz and Zhang [25], but with the required generality for handling general strongly convex regularizers and smoothness/Lipschitzness with respect to general norms.

We prove the theorems for running Prox-SDCA while choosing $\Delta \alpha_{i}$ as in Option I. A careful examination of the proof easily reveals that the results hold for the other options as well. More specifically, Lemma 6 only requires choosing $\Delta \alpha_{i}=s\left(u_{i}^{(t-1)}-\alpha_{i}^{(t-1)}\right)$ as in (23), and Option III chooses $s$ to optimize the bound on the right hand side of (25), and hence ensures that the choice can do no worse than the result of Lemma 6 with any $s$. The simplification in Option IV and V employs the specific simplification of the bound in Lemma 6 in the proof of the theorems.

The key lemma is the following:

Lemma 6. Assume that $\phi_{i}^{*}$ is $\gamma$-strongly-convex. For any iteration $t$, let $\mathbb{E}_{t}$ denote the expectation with respect to the randomness in choosing $i$ at round $t$, conditional on the value of $\alpha^{(t-1)}$. Then, for any iteration $t$ and any $s \in[0,1]$ we have

$$
\mathbb{E}_{t}\left[D\left(\alpha^{(t)}\right)-D\left(\alpha^{(t-1)}\right)\right] \geq \frac{s}{n}\left[P\left(w^{(t-1)}\right)-D\left(\alpha^{(t-1)}\right)\right]-\left(\frac{s}{n}\right)^{2} \frac{G^{(t)}}{2 \lambda},
$$

where

$$
G^{(t)}=\frac{1}{n} \sum_{i=1}^{n}\left(\left\|X_{i}\right\|_{D \rightarrow D^{\prime}}^{2}-\frac{\gamma(1-s) \lambda n}{s}\right) \mathbb{E}_{t}\left[\left\|u_{i}^{(t-1)}-\alpha_{i}^{(t-1)}\right\|_{D}^{2}\right]
$$

and $-u_{i}^{(t-1)}=\nabla \phi_{i}\left(X_{i}^{\top} w^{(t-1)}\right)$.

Proof. Since only the $i$ 'th element of $\alpha$ is updated, the improvement in the dual objective can be written as

$$
\begin{aligned}
& n\left[D\left(\alpha^{(t)}\right)-D\left(\alpha^{(t-1)}\right)\right] \\
= & \left(-\phi^{*}\left(-\alpha_{i}^{(t)}\right)-\lambda n g^{*}\left(v^{(t-1)}+(\lambda n)^{-1} X_{i} \Delta \alpha_{i}\right)\right)-\left(-\phi^{*}\left(-\alpha_{i}^{(t-1)}\right)-\lambda n g^{*}\left(v^{(t-1)}\right)\right)
\end{aligned}
$$

The smoothness of $g^{*}$ implies that $g^{*}(v+\Delta v) \leq h(v ; \Delta v)$, where $h(v ; \Delta v):=g^{*}(v)+\nabla g^{*}(v)^{\top} \Delta v+$ $\frac{1}{2}\|\Delta v\|_{D^{\prime}}^{2}$. Therefore,

$$
\begin{aligned}
& n\left[D\left(\alpha^{(t)}\right)-D\left(\alpha^{(t-1)}\right)\right] \\
\geq & \underbrace{\left(-\phi^{*}\left(-\alpha_{i}^{(t)}\right)-\lambda n h\left(v^{(t-1)} ;(\lambda n)^{-1} X_{i} \Delta \alpha_{i}\right)\right)}_{A}-\underbrace{\left(-\phi^{*}\left(-\alpha_{i}^{(t-1)}\right)-\lambda n g^{*}\left(v^{(t-1)}\right)\right)}_{B} .
\end{aligned}
$$


By the definition of the update we have for all $s \in[0,1]$ that

$$
\begin{aligned}
A & =\max _{\Delta \alpha_{i}}-\phi^{*}\left(-\left(\alpha_{i}^{(t-1)}+\Delta \alpha_{i}\right)\right)-\lambda n h\left(v^{(t-1)} ;(\lambda n)^{-1} X_{i} \Delta \alpha_{i}\right) \\
& \geq-\phi^{*}\left(-\left(\alpha_{i}^{(t-1)}+s\left(u_{i}^{(t-1)}-\alpha_{i}^{(t-1)}\right)\right)\right)-\lambda n h\left(v^{(t-1)} ;(\lambda n)^{-1} s X_{i}\left(u_{i}^{(t-1)}-\alpha_{i}^{(t-1)}\right)\right) .
\end{aligned}
$$

From now on, we omit the superscripts and subscripts. Since $\phi^{*}$ is $\gamma$-strongly convex, we have that

$$
\phi^{*}(-(\alpha+s(u-\alpha)))=\phi^{*}(s(-u)+(1-s)(-\alpha)) \leq s \phi^{*}(-u)+(1-s) \phi^{*}(-\alpha)-\frac{\gamma}{2} s(1-s)\|u-\alpha\|_{D}^{2}
$$

Combining this with 23 and rearranging terms we obtain that

$$
\begin{aligned}
A \geq-s \phi^{*}(-u)-(1-s) \phi^{*}(-\alpha)+\frac{\gamma}{2} s(1-s)\|u-\alpha\|_{D}^{2}-\lambda n h\left(v ;(\lambda n)^{-1} s X(u-\alpha)\right) \\
=-s \phi^{*}(-u)-(1-s) \phi^{*}(-\alpha)+\frac{\gamma}{2} s(1-s)\|u-\alpha\|_{D}^{2}-\lambda n g^{*}(v)-s w^{\top} X(u-\alpha)-\frac{s^{2}\|X(u-\alpha)\|_{D^{\prime}}^{2}}{2 \lambda n} \\
\geq-s\left(\phi^{*}(-u)+w^{\top} X u\right)+\left(-\phi^{*}(-\alpha)-\lambda n g^{*}(v)\right) \\
\quad+\frac{s}{2}\left(\gamma(1-s)-\frac{\left.s\|X\|_{D \rightarrow D^{\prime}}^{2}\right)\|u-\alpha\|_{D}^{2}+s\left(\phi^{*}(-\alpha)+w^{\top} X \alpha\right) .}{\lambda n}\right.
\end{aligned}
$$

Since $-u=\nabla \phi\left(X^{\top} w\right)$ we have $\phi^{*}(-u)+w^{\top} X u=-\phi\left(X^{\top} w\right)$, which yields

$$
A-B \geq s\left[\phi\left(X^{\top} w\right)+\phi^{*}(-\alpha)+w^{\top} X \alpha+\left(\frac{\gamma(1-s)}{2}-\frac{s\|X\|_{D \rightarrow D^{\prime}}^{2}}{2 \lambda n}\right)\|u-\alpha\|_{D}^{2}\right] .
$$

Next note that with $w=\nabla g^{*}(v)$, we have $g(w)+g^{*}(v)=w^{\top} v$. Therefore:

$$
\begin{aligned}
P(w)-D(\alpha) & =\frac{1}{n} \sum_{i=1}^{n} \phi_{i}\left(X_{i}^{\top} w\right)+\lambda g(w)-\left(-\frac{1}{n} \sum_{i=1}^{n} \phi_{i}^{*}\left(-\alpha_{i}\right)-\lambda g^{*}(v)\right) \\
& =\frac{1}{n} \sum_{i=1}^{n} \phi_{i}\left(X_{i}^{\top} w\right)+\frac{1}{n} \sum_{i=1}^{n} \phi_{i}^{*}\left(-\alpha_{i}\right)+\lambda w^{\top} v \\
& =\frac{1}{n} \sum_{i=1}^{n}\left(\phi_{i}\left(X_{i}^{\top} w\right)+\phi_{i}^{*}\left(-\alpha_{i}\right)+w^{\top} X_{i} \alpha_{i}\right) .
\end{aligned}
$$

Therefore, if we take expectation of 25] w.r.t. the choice of $i$ we obtain that

$$
\frac{1}{s} \mathbb{E}_{t}[A-B] \geq[P(w)-D(\alpha)]-\frac{s}{2 \lambda n} \cdot \underbrace{\frac{1}{n} \sum_{i=1}^{n}\left(\left\|X_{i}\right\|_{D \rightarrow D^{\prime}}^{2}-\frac{\gamma(1-s) \lambda n}{s}\right) \mathbb{E}_{t}\left[\left\|u_{i}-\alpha_{i}\right\|_{D}^{2}\right]}_{=G^{(t)}} .
$$

We have obtained that

$$
\frac{n}{s} \mathbb{E}_{t}\left[D\left(\alpha^{(t)}\right)-D\left(\alpha^{(t-1)}\right)\right] \geq\left[P\left(w^{(t-1)}\right)-D\left(\alpha^{(t-1)}\right)\right]-\frac{s G^{(t)}}{2 \lambda n} .
$$

Multiplying both sides by $s / n$ concludes the proof of the lemma. 
Equipped with the above lemmas we are ready to prove Theorem 1 and Theorem 2 .

Proof of Theorem 1$]$ The assumption that $\phi_{i}$ is $(1 / \gamma)$-smooth implies that $\phi_{i}^{*}$ is $\gamma$-strongly-convex. We will apply Lemma 6 with

$$
s=\frac{n}{n+R^{2} /(\lambda \gamma)}=\frac{\lambda n \gamma}{R^{2}+\lambda n \gamma} \in[0,1] .
$$

Recall that $\left\|X_{i}\right\|_{D \rightarrow D^{\prime}} \leq R$. Therefore, the choice of $s$ implies that

$$
\left\|X_{i}\right\|_{D \rightarrow D^{\prime}}^{2}-\frac{\gamma(1-s) \lambda n}{s} \leq R^{2}-\frac{1-s}{s /(\lambda n \gamma)}=R^{2}-R^{2}=0
$$

and hence $G^{(t)} \leq 0$ for all $t$. This yields,

$$
\mathbb{E}_{t}\left[D\left(\alpha^{(t)}\right)-D\left(\alpha^{(t-1)}\right)\right] \geq \frac{s}{n}\left(P\left(w^{(t-1)}\right)-D\left(\alpha^{(t-1)}\right)\right) .
$$

Taking expectation of both sides with respect to the randomness at previous rounds, and using the law of total expectation, we obtain that

$$
\mathbb{E}\left[D\left(\alpha^{(t)}\right)-D\left(\alpha^{(t-1)}\right)\right] \geq \frac{s}{n} \mathbb{E}\left[P\left(w^{(t-1)}\right)-D\left(\alpha^{(t-1)}\right)\right] .
$$

But since $\epsilon_{D}^{(t-1)}:=D\left(\alpha^{*}\right)-D\left(\alpha^{(t-1)}\right) \leq P\left(w^{(t-1)}\right)-D\left(\alpha^{(t-1)}\right)$ and $D\left(\alpha^{(t)}\right)-D\left(\alpha^{(t-1)}\right)=\epsilon_{D}^{(t-1)}-\epsilon_{D}^{(t)}$, we obtain that

$$
\mathbb{E}\left[\epsilon_{D}^{(t)}\right] \leq\left(1-\frac{s}{n}\right) \mathbb{E}\left[\epsilon_{D}^{(t-1)}\right] \leq\left(1-\frac{s}{n}\right)^{t} \epsilon_{D}^{(0)} \leq \epsilon_{D}^{(0)} e^{-\frac{s t}{n}} .
$$

Therefore, whenever

$$
t \geq \frac{n}{s} \log \left(\epsilon_{D}^{(0)} / \epsilon_{D}\right)=\left(n+\frac{R^{2}}{\lambda \gamma}\right) \log \left(\epsilon_{D}^{(0)} / \epsilon_{D}\right),
$$

we are guaranteed that $\mathbb{E}\left[\epsilon_{D}^{(t)}\right]$ would be smaller than $\epsilon_{D}$.

Using again (28), we can also obtain that

$$
\mathbb{E}\left[P\left(w^{(t)}\right)-D\left(\alpha^{(t)}\right)\right] \leq \frac{n}{s} \mathbb{E}\left[D\left(\alpha^{(t+1)}\right)-D\left(\alpha^{(t)}\right)\right]=\frac{n}{s} \mathbb{E}\left[\epsilon_{D}^{(t)}-\epsilon_{D}^{(t+1)}\right] \leq \frac{n}{s} \mathbb{E}\left[\epsilon_{D}^{(t)}\right] .
$$

So, requiring $\mathbb{E}\left[\epsilon_{D}^{(t)}\right] \leq \frac{s}{n} \epsilon_{P}$ we obtain an expected duality gap of at most $\epsilon_{P}$. This means that we should require

$$
t \geq\left(n+\frac{R^{2}}{\lambda \gamma}\right) \log \left(\left(n+\frac{R^{2}}{\lambda \gamma}\right) \cdot \frac{\epsilon_{D}^{(0)}}{\epsilon_{P}}\right),
$$

which proves the first part of Theorem 1 .

Next, we sum the first inequality of (29) over $t=T_{0}+1, \ldots, T$ to obtain

$$
\mathbb{E}\left[\frac{1}{T-T_{0}} \sum_{t=T_{0}+1}^{T}\left(P\left(w^{(t)}\right)-D\left(\alpha^{(t)}\right)\right)\right] \leq \frac{n}{s\left(T-T_{0}\right)} \mathbb{E}\left[D\left(\alpha^{(T+1)}\right)-D\left(\alpha^{\left(T_{0}+1\right)}\right)\right] .
$$

Now, if we choose $\bar{w}, \bar{\alpha}$ to be either the average vectors or a randomly chosen vector over $t \in\left\{T_{0}+\right.$ $1, \ldots, T\}$, then the above implies

$$
\begin{aligned}
\mathbb{E}[P(\bar{w})-D(\bar{\alpha})] & \leq \frac{n}{s\left(T-T_{0}\right)} \mathbb{E}\left[D\left(\alpha^{(T+1)}\right)-D\left(\alpha^{\left(T_{0}+1\right)}\right)\right] \\
& \left.\leq \frac{n}{s\left(T-T_{0}\right)} \mathbb{E}\left[\epsilon_{D}^{\left(T_{0}+1\right)}\right)\right] \\
& \leq \frac{n}{s\left(T-T_{0}\right)} \epsilon_{D}^{(0)} e^{-\frac{s T_{0}}{n}}
\end{aligned}
$$


It follows that in order to obtain a result of $\mathbb{E}[P(\bar{w})-D(\bar{\alpha})] \leq \epsilon_{P}$, we need to have

$$
T_{0} \geq \frac{n}{s} \log \left(\frac{n \epsilon_{D}^{(0)}}{s\left(T-T_{0}\right) \epsilon_{P}}\right) .
$$

In particular, the choice of $T-T_{0}=\frac{n}{s}$ and $T_{0}=\frac{n}{s} \log \left(\epsilon_{D}^{(0)} / \epsilon_{P}\right)$ satisfies the above requirement.

Proof of Theorem 2 Define $t_{0}=\left\lceil\frac{n}{s} \log \left(2 \epsilon_{D}^{(0)} / \epsilon_{D}\right)\right\rceil$. The proof of Theorem 1 implies that for every $t$, $\mathbb{E}\left[\epsilon_{D}^{(t)}\right] \leq \epsilon_{D}^{(0)} e^{-\frac{s t}{n}}$. By Markov's inequality, with probability of at least $1 / 2$ we have $\epsilon_{D}^{(t)} \leq 2 \epsilon_{D}^{(0)} e^{-\frac{s t}{n}}$. Applying it for $t=t_{0}$ we get that $\epsilon_{D}^{\left(t_{0}\right)} \leq \epsilon_{D}$ with probability of at least $1 / 2$. Now, lets apply the same argument again, this time with the initial dual sub-optimality being $\epsilon_{D}^{\left(t_{0}\right)}$. Since the dual is monotonically non-increasing, we have that $\epsilon_{D}^{\left(t_{0}\right)} \leq \epsilon_{D}^{(0)}$. Therefore, the same argument tells us that with probability of at least $1 / 2$ we would have that $\epsilon_{D}^{\left(2 t_{0}\right)} \leq \epsilon_{D}$. Repeating this $\left\lceil\log _{2}(1 / \delta)\right\rceil$ times, we obtain that with probability of at least $1-\delta$, for some $k$ we have that $\epsilon_{D}^{\left(k t_{0}\right)} \leq \epsilon_{D}$. Since the dual is monotonically non-decreasing, the claim about the dual sub-optimality follows.

Next, for the duality gap, using (27) we have that for every $t$ such that $\epsilon_{D}^{(t-1)} \leq \epsilon_{D}$ we have

$$
P\left(w^{(t-1)}\right)-D\left(\alpha^{(t-1)}\right) \leq \frac{n}{s} \mathbb{E}\left[D\left(\alpha^{(t)}\right)-D\left(\alpha^{(t-1)}\right)\right] \leq \frac{n}{s} \epsilon_{D} .
$$

This proves the second claim of Theorem 2

For the last claim, suppose that at round $T_{0}$ we have $\epsilon_{D}^{\left(T_{0}\right)} \leq \epsilon_{D}$. Let $T=T_{0}+n / s$. It follows that if we choose $t$ uniformly at random from $\left\{T_{0}, \ldots, T-1\right\}$, then $\mathbb{E}\left[P\left(w^{(t)}\right)-D\left(\alpha^{(t)}\right)\right] \leq \epsilon_{D}$. By Markov's inequality, with probability of at least $1 / 2$ we have $P\left(w^{(t)}\right)-D\left(\alpha^{(t)}\right) \leq 2 \epsilon_{D}$. Therefore, if we choose $\log _{2}(2 / \delta)$ such random $t$, with probability $\geq 1-\delta / 2$, at least one of them will have $P\left(w^{(t)}\right)-D\left(\alpha^{(t)}\right) \leq$ $2 \epsilon_{D}$. Combining with the first claim of the theorem, choosing $\epsilon_{D}=\epsilon_{P} / 2$, and applying the union bound, we conclude the proof of the last claim of Theorem 2 .

\section{References}

[1] Michel Baes. Estimate sequence methods: extensions and approximations. Institute for Operations Research, ETH, Zürich, Switzerland, 2009.

[2] A. Beck and M. Teboulle. A fast iterative shrinkage-thresholding algorithm for linear inverse problems. SIAM Journal on Imaging Sciences, 2(1):183-202, 2009.

[3] M. Collins, A. Globerson, T. Koo, X. Carreras, and P. Bartlett. Exponentiated gradient algorithms for conditional random fields and max-margin markov networks. Journal of Machine Learning Research, 9:1775-1822, 2008.

[4] Andrew Cotter, Ohad Shamir, Nathan Srebro, and Karthik Sridharan. Better mini-batch algorithms via accelerated gradient methods. arXiv preprint arXiv:1106.4574, 2011.

[5] K. Crammer and Y. Singer. On the algorithmic implementation of multiclass kernel-based vector machines. Journal of Machine Learning Research, 2:265-292, 2001. 
[6] Alexandre d'Aspremont. Smooth optimization with approximate gradient. SIAM Journal on Optimization, 19(3):1171-1183, 2008.

[7] Olivier Devolder, Francois Glineur, and Yu. Nesterov. First-order methods of smooth convex optimization with inexact oracle. Technical Report 2011/2, CORE, 2011. available: http://www.uclouvain.be/cps/ucl/doc/core/documents/coredp2011_2web.pdf.

[8] J. Duchi and Y. Singer. Efficient online and batch learning using forward backward splitting. The Journal of Machine Learning Research, 10:2899-2934, 2009.

[9] John Duchi, Shai Shalev-Shwartz, Yoram Singer, and Tushar Chandra. Efficient projections onto the 1 1-ball for learning in high dimensions. In Proceedings of the 25th international conference on Machine learning, pages 272-279. ACM, 2008.

[10] John Duchi, Shai Shalev-Shwartz, Yoram Singer, and Ambuj Tewari. Composite objective mirror descent. In Proceedings of the 23rd Annual Conference on Learning Theory, pages 14-26, 2010.

[11] Saeed Ghadimi and Guanghui Lan. Optimal stochastic approximation algorithms for strongly convex stochastic composite optimization i: A generic algorithmic framework. SIAM Journal on Optimization, 22(4):1469-1492, 2012.

[12] Chonghai Hu, Weike Pan, and James T Kwok. Accelerated gradient methods for stochastic optimization and online learning. In Advances in Neural Information Processing Systems, pages 781-789, 2009.

[13] S. Lacoste-Julien, M. Jaggi, M. Schmidt, and P. Pletscher. Stochastic block-coordinate frank-wolfe optimization for structural svms. arXiv preprint arXiv:1207.4747, 2012.

[14] J. Langford, L. Li, and T. Zhang. Sparse online learning via truncated gradient. In NIPS, pages 905-912, 2009.

[15] Nicolas Le Roux, Mark Schmidt, and Francis Bach. A Stochastic Gradient Method with an Exponential Convergence Rate for Strongly-Convex Optimization with Finite Training Sets. arXiv preprint arXiv:1202.6258, 2012.

[16] Y. Nesterov. Efficiency of coordinate descent methods on huge-scale optimization problems. SIAM Journal on Optimization, 22(2):341-362, 2012.

[17] Yurii Nesterov. Smooth minimization of non-smooth functions. Mathematical Programming, 103(1): 127-152, 2005.

[18] Yurii Nesterov. Gradient methods for minimizing composite objective function, 2007.

[19] Peter Richtárik and Martin Takáč. Iteration complexity of randomized block-coordinate descent methods for minimizing a composite function. Mathematical Programming, pages 1-38, 2012.

[20] Mark Schmidt, Nicolas Le Roux, and Francis Bach. Convergence rates of inexact proximal-gradient methods for convex optimization. Technical Report arXiv:1109.2415, arXiv, 2011.

[21] S. Shalev-Shwartz and A. Tewari. Stochastic methods for 1 1-regularized loss minimization. The Journal of Machine Learning Research, 12:1865-1892, 2011. 
[22] S. Shalev-Shwartz, Y. Singer, and N. Srebro. Pegasos: Primal Estimated sub-GrAdient SOlver for SVM. In ICML, pages 807-814, 2007.

[23] Shai Shalev-Shwartz and Ambuj Tewari. Stochastic methods for $1_{1}$ regularized loss minimization. In ICML, page 117, 2009.

[24] Shai Shalev-Shwartz and Tong Zhang. Stochastic dual coordinate ascent methods for regularized loss minimization. arXiv preprint arXiv:1209.1873, 2012.

[25] Shai Shalev-Shwartz and Tong Zhang. Stochastic dual coordinate ascent methods for regularized loss minimization. Journal of Machine Learning Research, 14:567-599, Feb 2013.

[26] Shai Shalev-Shwartz, Nathan Srebro, and Tong Zhang. Trading accuracy for sparsity in optimization problems with sparsity constraints. SIAM Journal on Optimization, 20(6):2807-2832, 2010.

[27] Lin Xiao. Dual averaging method for regularized stochastic learning and online optimization. Journal of Machine Learning Research, 11:2543-2596, 2010.

[28] Tong Zhang. On the dual formulation of regularized linear systems. Machine Learning, 46:91-129, 2002. 